\title{
Enhanced triacylglycerol catabolism by carboxylesterase 1 promotes aggressive colorectal carcinoma
}

\author{
Daria Capece, ${ }^{1,2}$ Daniel D’Andrea, ${ }^{1}$ Federica Begalli, ${ }^{1}$ Laura Coracci, ${ }^{3}$ Laura Tornatore, ${ }^{1}$ James L. Alexander, ${ }^{4}$ \\ Alessandra Di Veroli, ${ }^{3}$ Shi-Chi Leow, ${ }^{5}$ Thamil S. Vaiyapuri, ${ }^{6}$ James K. Ellis, ${ }^{1,7}$ Daniela Verzella, Jason Bennett, ${ }^{1}$ Luca Savino, ${ }^{1,8}$ \\ Yue Ma, ${ }^{4}$ James S. McKenzie, ${ }^{7}$ Maria Luisa Doria, ${ }^{7}$ Sam E. Mason, ${ }^{7}$ Kern Rei Chng, ${ }^{9}$ Hector C. Keun, ${ }^{7}$ Cary Frost, ${ }^{4}$ \\ Vinay Tergaonkar, ${ }^{6}$ Katarzyna Broniowska, ${ }^{10}$ Walter Stunkel, ${ }^{5}$ Zoltan Takats, ${ }^{7}$ James M. Kinross, ${ }^{7}$ Gabriele Cruciani, ${ }^{3}$ \\ and Guido Franzoso ${ }^{1}$
}

\begin{abstract}
'Department of Immunology and Inflammation, Imperial College London, London, United Kingdom. ²Department of Biotechnological and Applied Clinical Sciences (DISCAB), University of L'Aquila, L'Aquila, Italy. ${ }^{3}$ Department of Chemistry, Biology and Biotechnology, University of Perugia, Perugia, Italy. ${ }^{4}$ Department of Metabolism, Digestion and Reproduction, Imperial College London, London, United Kingdom. ${ }^{5}$ Singapore Institute for Clinical Sciences (SICS), and ${ }^{6}$ Institute of Molecular and Cell Biology (IMCB), Agency for Science Technology and Research (A*STAR), Singapore. ${ }^{7}$ Department of Surgery and Cancer, Imperial College London, London, United Kingdom. ${ }^{8}$ Department of Medical, Oral, and Biotechnological Sciences, “C. D’Annunzio" University of Chieti-Pescara, Chieti, Italy. ${ }^{9} \mathrm{Cenome}$ Institute of Singapore, Singapore. ${ }^{10}$ Metabolon, Inc., Morrisville, North Carolina, USA.
\end{abstract}

The ability to adapt to low-nutrient microenvironments is essential for tumor cell survival and progression in solid cancers, such as colorectal carcinoma (CRC). Signaling by the NF-KB transcription factor pathway associates with advanced disease stages and shorter survival in patients with CRC. NF-אB has been shown to drive tumor-promoting inflammation, cancer cell survival, and intestinal epithelial cell (IEC) dedifferentiation in mouse models of CRC. However, whether NF- $\mathrm{BB}$ affects the metabolic adaptations that fuel aggressive disease in patients with CRC is unknown. Here, we identified carboxylesterase 1 (CES1) as an essential NF-кB-regulated lipase linking obesity-associated inflammation with fat metabolism and adaptation to energy stress in aggressive CRC. CES1 promoted CRC cell survival via cell-autonomous mechanisms that fuel fatty acid oxidation (FAO) and prevent the toxic build-up of triacylglycerols. We found that elevated CES1 expression correlated with worse outcomes in overweight patients with CRC. Accordingly, NF- $\mathrm{KB}$ drove CES1 expression in CRC consensus molecular subtype 4 (CMS4), which is associated with obesity, stemness, and inflammation. CES1 was also upregulated by gene amplifications of its transcriptional regulator HNF4A in CMS2 tumors, reinforcing its clinical relevance as a driver of CRC. This subtype-based distribution and unfavorable prognostic correlation distinguished CES1 from other intracellular triacylglycerol lipases and suggest CES1 could provide a route to treat aggressive CRC.

\section{Introduction}

Tumor cells must adapt to the low nutrient concentrations in the tumor microenvironment (TME) in order to survive, proliferate, and spread to distant sites (1-4). The availability of nutrients, such as glucose, is generally lower in solid tumors than in normal tissues because of the higher metabolic requirements of rapidly proliferating tumor cells and inadequate blood supply due to the abnormal and inefficient tumor vasculature $(5,6)$. This poses a major metabolic challenge for tumor cells, exacerbated by the presence of driver mutations that commit these cells to a biosynthetic metabolic program while restricting their capacity to respond to metabolic stress $(2,3)$. Tumor cell clones that are able to meet this chal-

Authorship note: DC and DD contributed equally to this work and are co-first authors. Conflict of interest: The authors have declared that no conflict of interest exists. Copyright: @ 2021, Capece et al. This is an open access article published under the terms of the Creative Commons Attribution 4.0 International License.

Submitted: March 9, 2020; Accepted: April 14, 2021; Published: June 1, 2021

Reference information: J Clin Invest. 2021;131(11):e137845.

https://doi.org/10.1172/JCl137845. lenge have a significant growth advantage and greater metabolic adaptability, which fuel malignant progression, epithelial to mesenchymal transition (EMT), and metastatic spread $(4,7)$.

Several adaptive metabolic mechanisms have been described in colorectal carcinoma (CRC) (8-12). Early studies have shown that glucose deprivation drives the acquisition of RAS-pathway mutations, which promote CRC cell adaptation to low glucose availability in part by increasing glucose uptake through the upregulation of glucose transporter 1 (GLUT1) (8). Subsequent studies have reported additional metabolic responses of CRC cells to starvation, including glucose deprivation-induced MYC stabilization, which enhances glycolytic flux by upregulating GLUT1 and other glycolysis-associated genes $(9,10)$, and alterations in the expression of genes involved in glutamine metabolism $(11,12)$. Recent reports have shown that metabolic adaptations also play an important role in the EMT and in metastatic dissemination, which requires CRC cells to migrate through and adapt to diverse metabolic tumor environments, both upon entering the circulation and colonizing distal sites $(4,7,13,14)$. Strong evidence also indicates that an upregulation of oxidative metabolism and fatty acid oxidation (FAO) during 

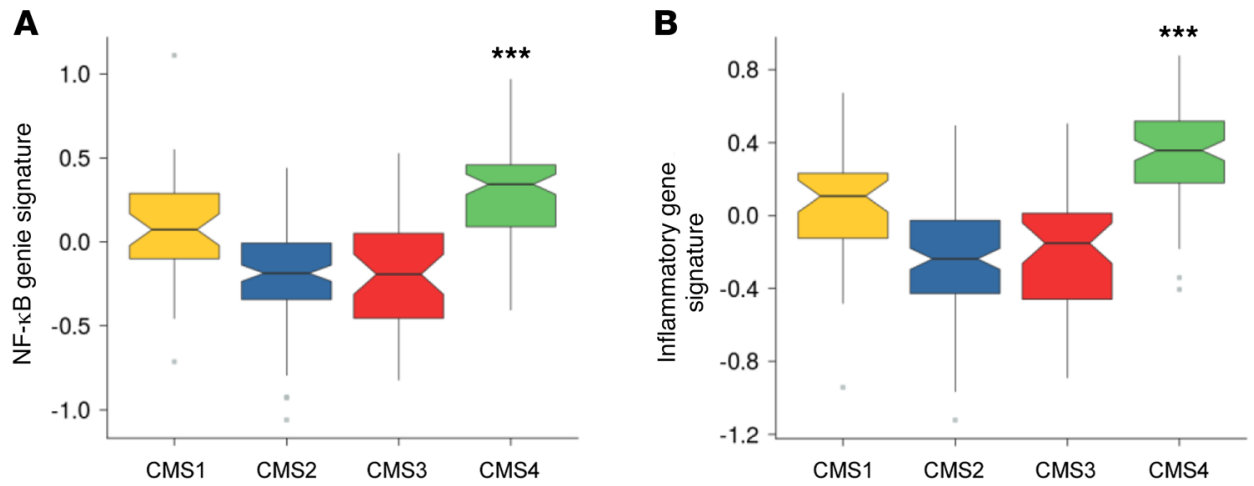

\section{C}
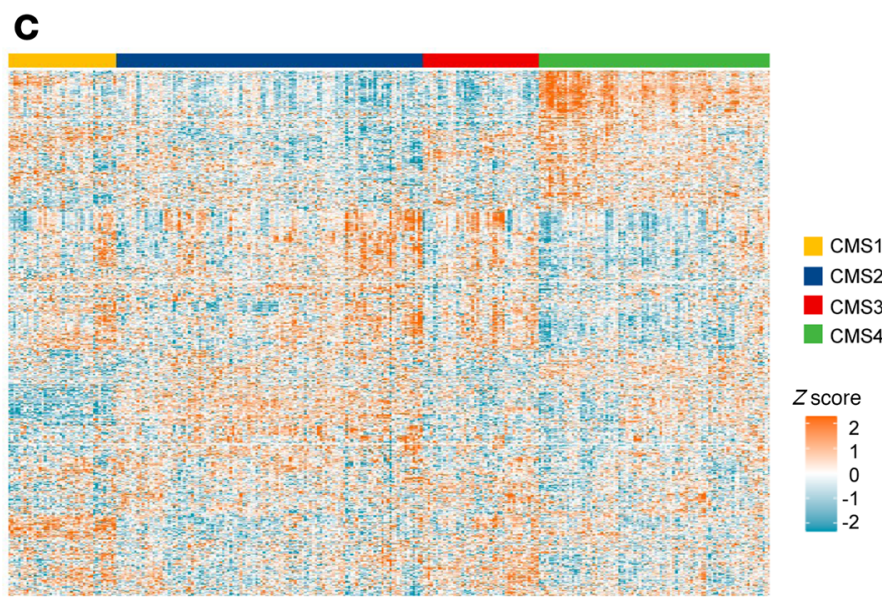

D

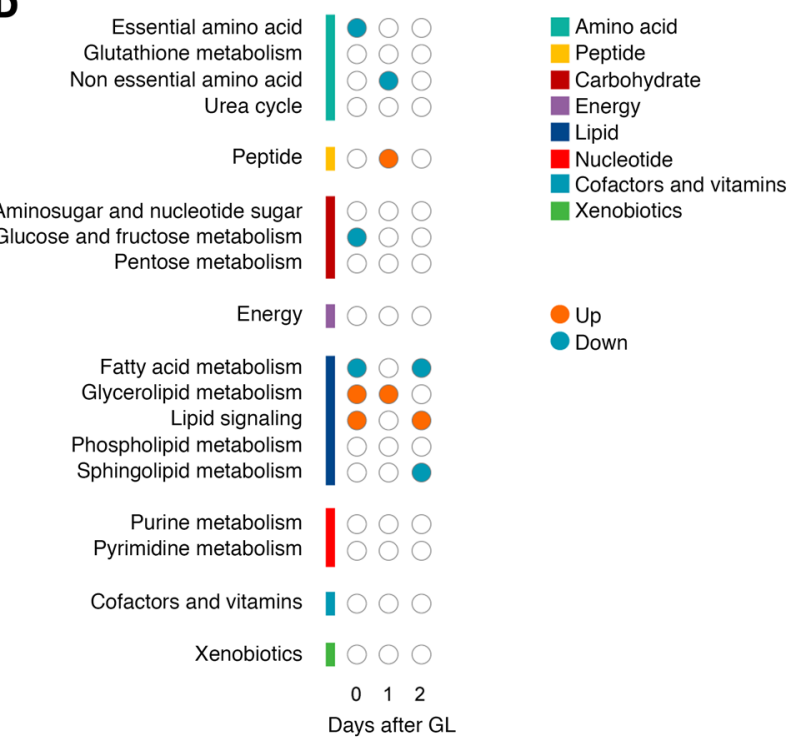

E

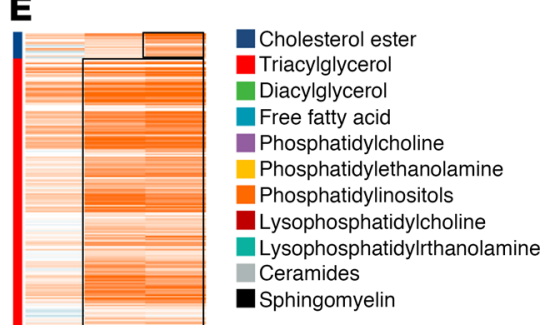


Figure 1. NF-KB is activated in the mesenchymal CMS4 CRC subtype and regulates lipid metabolism upon starvation. Box plots showing the median $z$ scores of the NF- $\mathrm{KB}$-activation (A) and inflammatory (B) gene signatures in each CMS subtype from patients with CRC $(n=296)$ in The Cancer Genome Atlas (TCGA) data set. Shown in the box plots are the median values (horizontal lines), 25th to 75 th percentiles (box outlines), and highest and lowest values within 1.5 times the interquartile range (vertical lines). Notches denote the $95 \%$ confidence interval of the medians. Samples from each CMS subtype were compared with other CRC samples by using 2-tailed Student's $t$ test. ${ }^{* *} P<0.001$. Statistical significance for multiple comparisons was calculated using the Kruskal-Wallis test $\left(P<2.2 \times 10^{-16}\right)$. (C) Hierarchical clustering of the 1795 metabolic genes present in the Reactome database from the patients in $\mathbf{A}$ and $\mathbf{B}$, arranged according to CMS subtype. The $z$ scores of gene expression are depicted as a gradient from azure (low expression) to orange (high expression). Yellow, CMS1; blue, CMS2; red, CMS3; green, CMS4. (D) Metabolite set enrichment analysis of significantly deregulated biochemicals $(q<0.05)$ in MEFs expressing RelA-specific (RelA) relative to nonspecific (ns) shRNAs $(n=5)$, cultured under normal conditions $(0)$ or for the indicated times under $\mathrm{CL} . P$ values were corrected using the Benjamini-Hochberg false-discovery rate (FDR) procedure. Orange, overenrichment (up); azure, underenrichment (down). (E) Heatmap showing the $\log _{2}$ fold change of metabolic species in the indicated lipid classes in RelA-deficient relative to control MEFs treated as in $\mathbf{D}$. Shown are the lipid species having a value different from 0 in at least 1 of the time points investigated. Orange, increased abundance; azure, decreased abundance. Framed in black are the lipid classes showing statistically significant accumulation, as determined by a hypergeometric test, corrected using the Benjamini-Hochberg procedure (CE: $\mathrm{d} 2, q=4.55 \times$ $10^{-2}$; triacylglycerol: $\left.\mathrm{d} 1, q=4.44 \times 10^{-7} ; \mathrm{d} 2, q=1.19 \times 10^{-5}\right)$. For $\mathbf{D}$ and $\mathbf{E}$, metabolic analysis were conducted at Metabolon.

energy stress is essential for EMT, metastatic spread, and therapy resistance in many cancer types, including CRC (15-17). However, the precise mechanisms underpinning this metabolic plasticity of cancer cells and its influence on tumor behavior and clinical outcomes in patients with CRC remain poorly understood.

NF- $\mathrm{BB}$ transcription factors are central regulators of immunity and inflammation (18). They are also major drivers of oncogenesis, disease recurrence, and therapy resistance, owing to their capacity to upregulate genes that suppress cancer cell apoptosis and orchestrate inflammation in the $\operatorname{TME}(18,19)$. Studies in mouse models of CRC have demonstrated an essential role for NF- $\mathrm{KB}$ in both tumor initiation and progression $(20,21)$. During CRC initiation, NF-KB signaling enhances WNT/ $\beta$-catenin activation and stemness in intestinal epithelial cells (IECs) (20), whereas in established tumors, NF- $\mathrm{\kappa B}$ mediates a crucial link between malignancy and inflammation by stimulating tumor-promoting inflammation and the survival of intestinal tumor cells (21). Other reports have implicated NF- $\kappa B$ signaling in the regulation of energy metabolism, metabolic stress adaptation, and EMT in both models of inflammation and cancer, including CRC $(18,22-28)$. In keeping with these findings, several clinical studies have identified a correlation between higher NF- $\mathrm{kB}$ activity and shorter overall survival (OS), as well as more advanced disease stages, in patients with CRC (29-32). However, the paucity of the molecular clinical investigations has limited the mechanistic understanding of the pathogenic relationship of NF- $\mathrm{\kappa B}$ signaling with tumor phenotype and clinical evolution in CRC. Consequently, precisely how NF- $\mathrm{\kappa B}$ activation drives aggressive disease progression in patients with $\mathrm{CRC}$ remains poorly understood.
The consensus molecular subtype (CMS) classification of CRC recently provided a robust molecular subtyping system for $\mathrm{CRC}$ and an important new tool for deciphering how NF- $\mathrm{\kappa B}$ impacts CRC evolution and clinical outcomes (33). Thus, we sought to interrogate this classification system as a starting point to investigate whether and how NF- $\mathrm{kB}$ activity relates to intrinsic core biologic underpinnings of human CRC and features previously associated with NF-kB-driven colorectal tumors in mouse models. We reasoned that this approach might reveal novel core cancer cell vulnerabilities that could provide better treatments for patients with CRC who are affected by hard-to-treat forms of the disease. Using this approach, we identify carboxylesterase 1 (CES1) as an essential NF-kB-regulated lipase promoting cancer cell survival and adaptation to energy stress conditions in aggressive CRC. We found that elevated CES1 expression correlates with worse prognosis in overweight patients with CRC and is enriched downstream of NF- $\mathrm{KB}$ in mesenchymal consensus molecular subtype 4 (CMS4), which is associated with stemness, obesity, and inflammation. Our results uncover a metabolic oncogenic pathway that is mediated by NF- $\mathrm{kB}$ via CES1-dependent lipid catabolism, revealing an intriguing association of NF- $\mathrm{\kappa B}$ signaling and inflammation with obesity, fat catabolism, and metabolic adaptation in aggressive CMS4 tumors. These results underscore the clinical relevance of CES1 as a central driver of CRC and suggest CES1 could provide an effective route to treat patients with CRC with particularly poor prognosis.

\section{Results}

Inflammation and increased NF- $\kappa B$ activity cosegregate with the aggressive mesenchymal CMS4 subtype. Our analysis of human data sets demonstrated that the NF- $\mathrm{\kappa B}$ activation and inflammatory gene signatures are significantly upregulated in the mesenchymal CMS4 subtype of CRC (Figure 1, A and B), which is associated with stemness, EMT, TGF- $\beta$ activation, and shorter OS and relapse-free survival (33). This finding is consistent with the reported correlation between enhanced NF- $\mathrm{kB}$ activity and worse clinical outcomes in patients with CRC (29-32). CMS4 tumors also exhibited a distinctive pattern of metabolic gene expression compared with other CRC subtypes (Figure 1C). Since NF- $\kappa$ B is a transcriptional regulator of cell metabolism, in addition to being a central regulator of inflammation and the EMT $(18,19,22-28)$, this suggested that increased NF- $\mathrm{\kappa B}$ activation could contribute to the distinct metabolic features of CMS4 tumors.

$N F-\kappa B$ inhibition markedly disrupts lipid metabolism under energy stress conditions. To test this hypothesis, while excluding any potential confounding effects resulting from the idiosyncratic metabolic dependencies of cancer cells, we investigated the global impact of NF- $\mathrm{KB}$ inhibition on the metabolism of nontransformed mouse embryonic fibroblasts (MEFs) cultured under low glucose conditions (24), mimicking the low-glucose concentrations present in the tumor microenvironment (TME) $(5,6)$. As shown in Supplemental Figure 1, A-C (supplemental material available online with this article; https://doi.org/10.1172/JCI137845DS1), RNA interference-mediated (RNAi-mediated) inhibition of the dominant NF- $\mathrm{kB}$ subunit RelA $(18,20,34)$ altered the cellular abundance of over 430 metabolites, assessed by mass spectrometry (MS) before and/or after glucose limitation (GL) (Supplemental 
A

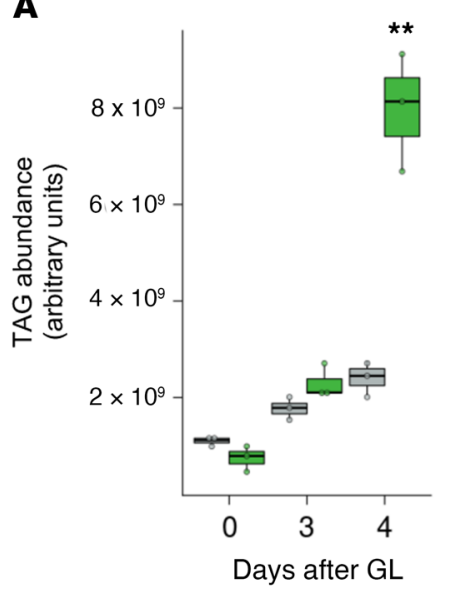

B

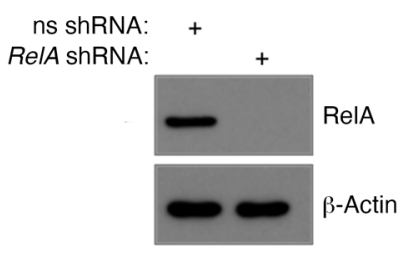

C

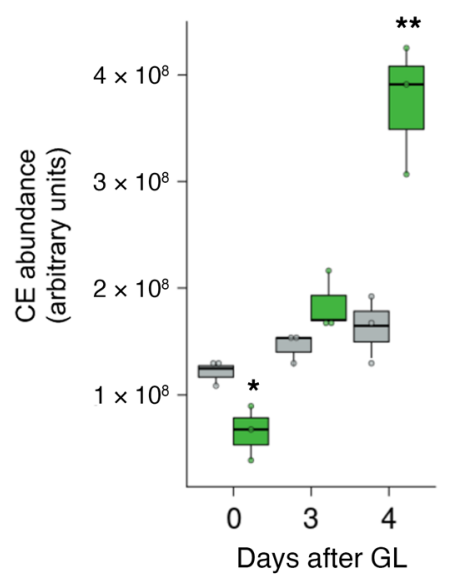

ns shRNA

RelA shRNA

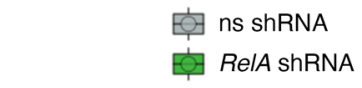

D

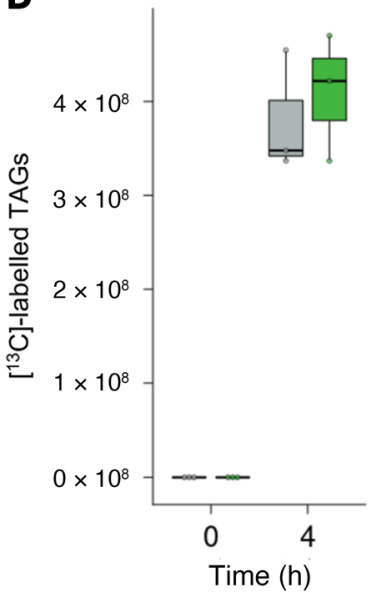

E

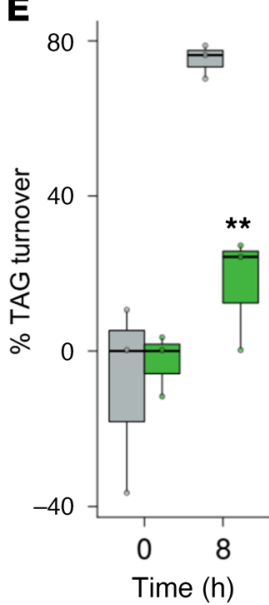

审 ns shRNA

RelA shRNA
$\mathbf{F}$

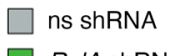

$\square$ RelA shRNA

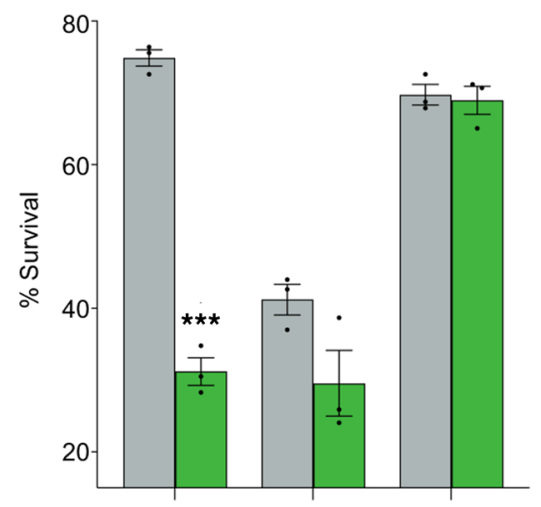

BSA

Oleate:

Vehicle:

Etomoxir:
审 ns shiRnA

EelA shRNA

G

$\square$ ns shRNA

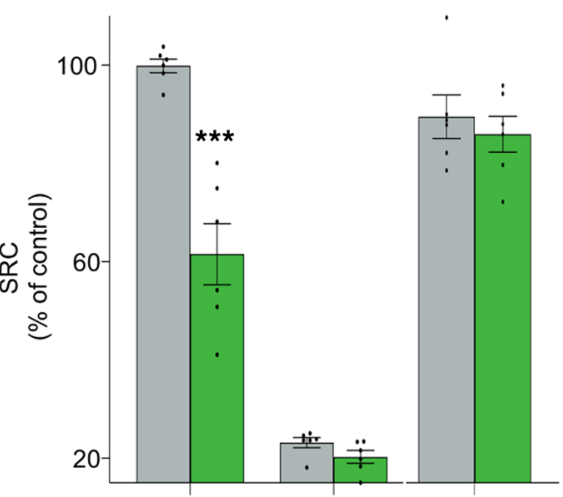

BSA:

Oleate:

Vehicle:

Etomoxir:
H

ns shRNA

RelA shRNA

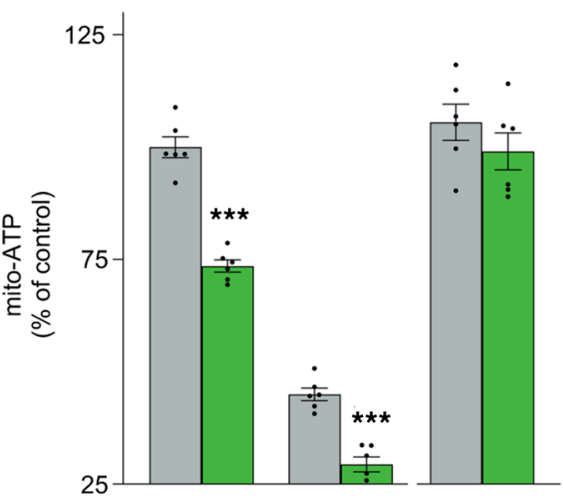

BSA

Oleate:

Vehicle:

Etomoxir: 
Figure 2. NF-kB inhibition impairs oxidative FFA metabolism and CRC cell survival during starvation. (A) Box plots showing the relative TAC abundance $(n=80)$ in CT-26 cells expressing RelA-specific versus ns shRNAs and cultured under normal (0) or GL conditions, as shown. The relative TAG abundance was derived by adding the peak area for each TAG, identified by LC-MS and Lipostar software (Molecular Discovery Ltd). (B) Western blots showing RelA and $\beta$-actin protein levels in CT-26 cells from A. (C) Box plots showing the relative CE abundance $(n=16)$ in CT-26 cells from $\mathbf{A}$ during $\mathrm{GL}$. The relative $\mathrm{CE}$ abundance was derived by adding the peak area for each CE, identified as in A. (D) Box plots showing the relative abundance of $\left[{ }^{3} \mathrm{C}\right]$-labeled TAGs $(n=7)$, determined by LC-MS flux analysis and Lipostar software in CT-26 cells expressing RelA-specific or ns shRNAs, before or after $\left[{ }^{[3} \mathrm{C}\right]$-oleate addition in the presence of $\mathrm{E} 600$ and etomoxir. (E) Box plots showing the TAC turnover calculated as in D, using $\left[{ }^{13} \mathrm{C}\right]$-oleate, in CT-26 cells expressing RelA-specific or ns shRNAs as in $\mathbf{A}$. TAG turnover reports the percentage of decrease of $\left[{ }^{13} \mathrm{C}\right]$-labeled TAC abundance $(n=40)$ at 8 relative to 0 hours, in the presence of triacsin C and forskolin. (F) Trypan blue exclusion assays showing the percentage of live CT-26 cells expressing RelA-specific or ns shRNAs as in $\mathbf{A}$ after 4 days under $\mathrm{GL}$, in the presence of the indicated treatments. Values denote mean \pm SD $(n=3)$. (G) SRC measured by Seahorse in cells from $\mathbf{A}$, treated as shown. (H) Mitochondrial ATP production rate (mito-ATP) measured by Seahorse in cells from $\mathbf{A}$, treated as shown. (A and $\mathbf{C}-\mathbf{E}$ ) Shown in the box plots are the medians (horizontal lines), 25th to 75th percentiles (box outlines), and highest and lowest values within 1.5 times the interquartile range (vertical lines). (F-H) BSA and BSA-oleate, $200 \mu \mathrm{M}$. (F and $\mathbf{G}$ ) Etomoxir, $10 \mu \mathrm{M}$. ( $\mathbf{G}$ and $\mathbf{H}$ ) Means report the percentages of the control values $\pm \operatorname{SEM}(n=6)$. (B and $\mathbf{F}-\mathbf{H})$ Experiments were conducted at least 3 times. (A and $\mathbf{C}-\mathbf{H}$ ) Statistical significance was calculated by 2-tailed Student's $t$ test. ${ }^{*} P<0.05 ;{ }^{* *} P<0.01$; ${ }^{* * *} P<0.001$.

Table 1). An enrichment analysis of these metabolites identified fatty acid metabolism, glycerolipid metabolism, and lipid signaling as the metabolic classes that were most significantly altered by RelA inhibition over time (Figure 1D).

To determine in more detail the impact of NF- $\mathrm{KB}$-dependent gene transcription on lipid metabolism and its possible involvement in the adaptation to low-glucose availability, we conducted an additional profiling of 1028 lipid species, using MEFs. As shown in Figure 1E, NF-kB/RelA knockdown affected metabolic species from multiple lipid classes, including phospholipids, lysophospholipids, and ceramides (Supplemental Table 1). However, the most profound effect of RelA inhibition was a significant enrichment of TAGs and, to a lesser extent, cholesteryl esters (CEs), with a marked accumulation of several TAG and CE species during GL. RelA depletion by RNAi similarly altered lipid metabolism in the CT-26 CRC cell line, resulting in an increased cellular content of both TAGs and CEs upon GL, and a lesser alteration of other lipid species, such as phosphocholines (Figure 2, A-C, Supplemental Figure 2A, and Supplemental Table 1) (35). The observed effects of RelA depletion on phospholipids and ceramides were in keeping with the NF-kB-dependent regulation of enzymes involved in inflammatory and stress-related lipid signaling, such as phospholipase A2 (36). However, the marked alteration of TAG and CE metabolism seen in RelA-deficient cells was not expected and suggested that NF- $\kappa B$ enhances lipolysis or reduces neutral lipid synthesis during low-glucose availability. To distinguish between these possibilities, we performed pulse-chase experiments using $\left[{ }^{13} \mathrm{C}\right]$-oleate and specific drug inhibitors to selectively block TAG hydrolysis or free-fatty acid (FFA) re-esterification. Interestingly, RNAi-mediated RelA inhibition had no effect on the rate of $\left[{ }^{13} \mathrm{C}\right]$-oleate incorporation into TAGs in CT-26 cells, indicating that NF- $\mathrm{BB}$ does not affect TAG synthesis or FFA cellular uptake (Figure 2D and Supplemental Table 1). In contrast, RelA deficiency markedly reduced TAG turnover during GL (Figure 2E and Supplemental Table 1), suggesting that NF- $\mathrm{kB}$ accelerates lipolysis.

$N F-\kappa B$ promotes energy homeostasis and CRC cell survival by increasing oxidative FFA metabolism during starvation. Cancer cells must adapt to low-glucose availability to survive in the poorly vascularized TME $(1-4,37)$. Thus, we investigated whether NF-кBdriven TAG catabolism could contribute to the metabolic adaptation of CRC cells to GL, as TAGs derived from lipid droplets are the preferred energy substrates used by cells under metabolic stress conditions $(3,38)$. RelA inhibition markedly diminished CT-26 cell survival during GL (Supplemental Figure 2B), in keeping with previous studies (24). However, treatment with BSA-bound oleate, which circumvents the requirement for lipolysis, completely rescued RelA-deficient cells from GL-induced death (Figure 2F). Furthermore, the cytotoxic effect of RelA depletion was recapitulated by the FAO inhibitor etomoxir, suggesting a role for FAO in the prosurvival function of NF- $\mathrm{BB}$ under GL (Figure 2F).

To clarify the mechanism by which NF- $\mathrm{KB}$ promotes cell survival during GL, we compared the mitochondrial bioenergetic profiles of RelA-deficient and control CT-26 cells. Notably, spare respiratory capacity (SRC), which reflects the respiratory reserve available for ATP production to support cell survival during energy stress conditions (39), was significantly reduced by RelA inhibition (Figure 2G and Supplemental Figure 2C). Treatment with etomoxir similarly diminished SRC in CT- 26 cells, abrogating the difference seen between RelA-deficient and control cells (Figure 2G and Supplemental Figure 2C). Mitochondrial ATP production was correspondingly impaired by the silencing of RelA (Figure $2 \mathrm{H}$ and Supplemental Figure 2D). Conversely, oleate supplementation reversed both the reduced mitochondrial ATP production rate and the reduced SRC observed in RelA-depleted cells. Together, these data demonstrate the importance of lipolysis in NF- $\mathrm{kB}-$ dependent metabolic homeostasis under GL conditions, while excluding a direct role for NF- $\mathrm{KB}$ in the modulation of FFA mitochondrial import or FAO (Figure 2, G and $\mathrm{H}$ ). We concluded that RelA deficiency reduces FFA availability for FAO and oxidative phosphorylation (OXPHOS) by impairing TAG hydrolysis under energy stress conditions, resulting in a metabolic crisis and cell death.

CES1 is a pivotal mediator of the effects of NF- $\kappa B$ on lipid catabolism. To understand how NF- $\mathrm{KB}$-dependent gene transcription regulated TAG catabolism, we analyzed the gene expression profile of RelA-deficient and control MEFs, at baseline and under GL, by using RNA sequencing. Among the 1792 metabolic genes listed in the Reactome database, we isolated the top 7 genes that were most markedly downregulated by RelA deficiency at all time points investigated (Figure 3A, Supplemental Figure 3A, and Supplemental Table 2). Notably, one of the genes in this group encoded carboxylesterase 1d (Ces1d), the murine ortholog of human CES1 (also known as triacylglycerol hydrolase), a TAG and CE lipase predominantly expressed in liver, adipose tissue, intestine, and lung (40). We confirmed by quantitative reverse transcription PCR (qRT-PCR) that Ces1d transcripts were strongly upregulated by GL in control MEFs, but only weakly in RelA-deficient MEFs (Supplemental Figure 3B), indicating that 
A

d0

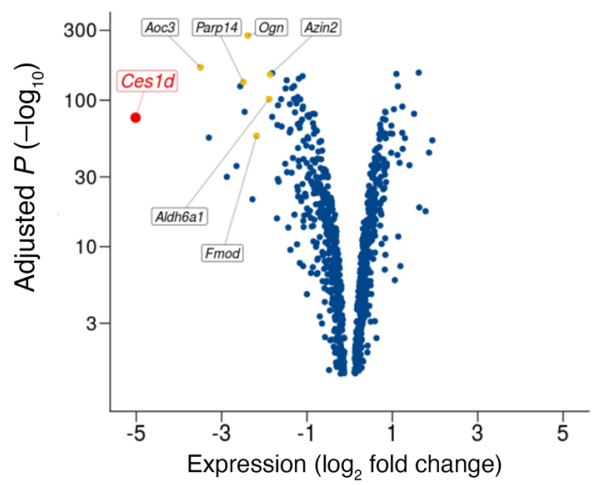

B

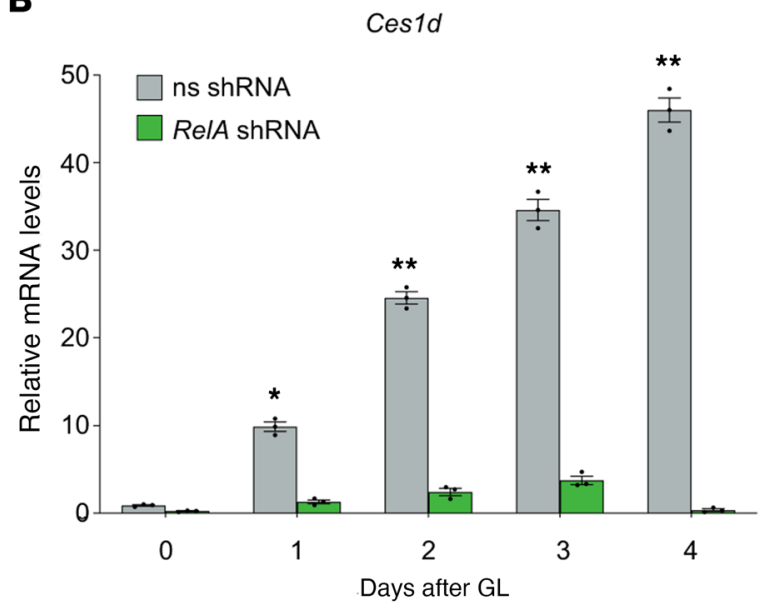

d2

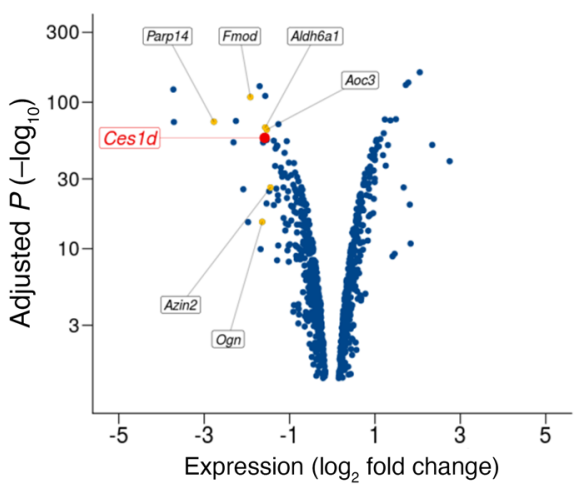

C

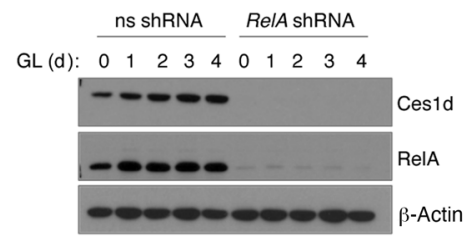

d3

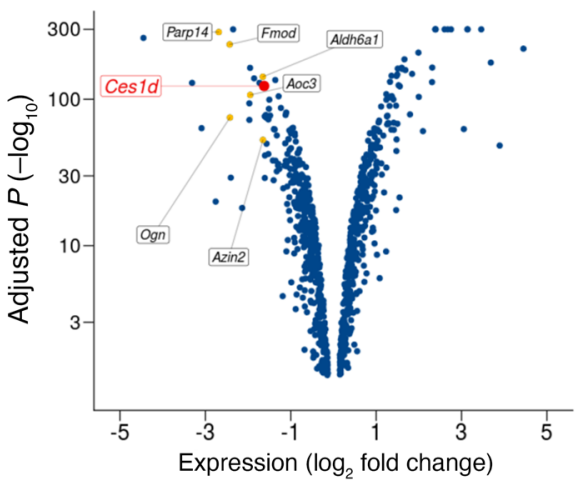

D

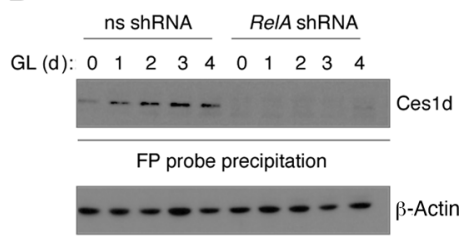

$\mathbf{E}$

$\square$ NF-kB binding site (кB) 1
$\square$ Control 1
$\square$ Control 2
$\square$ Control 3
$\square$ Control 4

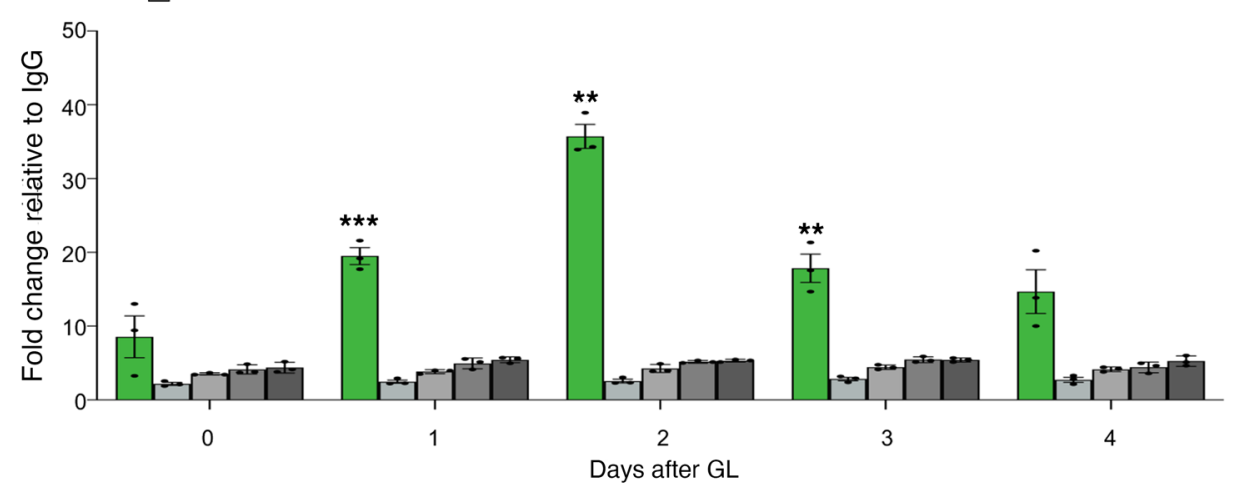

Figure 3. Ces1d is a direct transcriptional target of NF- $\mathrm{B}$ and one of the top 7 genes upregulated by NF- $\mathrm{B}$ during starvation. (A) Volcano plots showing differentially expressed metabolic genes ( $q<0.05 ; \mathrm{dO}, n=801 ; \mathrm{d} 2, n=726 ; \mathrm{d} 3, n=833$ ) in RelA-deficient relative to control MEFs $(n=5)$ cultured under normal conditions (d0) or for 2 (d2) or 3 (d3) days under $\mathrm{GL}$. Reported are the negative $\log _{10}$-transformed adjusted $P$ values plotted against the average $\log _{2}$ fold changes. Dots represent individual genes. Ces1d is depicted as a red dot; the other 6 most markedly downregulated metabolic genes in RelA-deficient relative to control MEFs, across all time points investigated, are depicted as orange dots. (B) qRT-PCR showing the Ces1d mRNA levels in CT-26 cells expressing RelA-specific or ns shRNAs and cultured under normal conditions (0) or for the indicated times under GL. Values denote mean \pm SD $(n=3)$. (C) Western blots showing the protein levels of Ces1d, RelA, and $\beta$-actin in CT-26 cells from B. (D) Desthiobiotin-fluorophosphonate activitybased (FP) probe precipitation assays showing the Ces1d-specific serine esterase activity in CT-26 cells from B, as determined by streptavidin-mediated precipitation of the biotin-bound FP probe followed by Western blots with anti-Ces1d antibody. $\beta$-actin in the total cell lysates (input) used for the activity-based protein profile (ABPP) assay is shown as a loading control. (E) Chromatin immunoprecipitation assays showing the binding of NF-KB/RelA complexes to $\kappa B$ DNA element 1 ( $\kappa B 1$ ) in the promoter region of Ces1d or the indicated control DNA regions (controls 1-4) in CT-26 cells from B. Values denote mean \pm SEM $(n=3)$. (B-E) Experiments were conducted at least 3 times. (B and E) Statistical significance was calculated by 2-tailed Student's $t$ test. ${ }^{*} P<0.05$; ${ }^{* *} P<0.01$; ${ }^{* *} P<0.001$. 
Ces1d expression depends on $\mathrm{NF}-\kappa \mathrm{B}$. The basal expression and upregulation of Ces1d transcript and protein levels in CT-26 CRC cells under GL also required NF- $\kappa \mathrm{B}$, as shown by RelA knockdown (Figure 3, B and C). Consistent with these results, RelA depletion by RNAi ablated Ces1d hydrolase activity, assessed by using a serine esterase activity-based probe (41), thus establishing the importance of NF- $\kappa \mathrm{B}$ in promoting the Ces1d enzymatic function in CRC cells (Figure 3D). In contrast, NF- $\kappa \mathrm{B}$ had no effect on Ser660-specific phosphorylation of hormone-sensitive lipase (Hsl) (Supplemental Figure 3C). Chromatin immunoprecipitation identified at least $10 \mathrm{NF}-\kappa \mathrm{B} /$ RelA-binding $(\kappa \mathrm{B})$ DNA elements in the promoter and intronic regions of the Ces1d gene (Supplemental Figure 3D). These DNA elements were frequently occupied by RelA-containing NF- $\kappa \mathrm{B}$ complexes at baseline, and their occupancy by NF- $\kappa$ B invariably increased upon GL (Figure $3 \mathrm{E}$ and Supplemental Figure 3E). Luciferase assays and mutational DNA analyses confirmed the functional relevance of at least 6 of these $\kappa \mathrm{B}$ elements for Ces1d transcription (Supplemental Figure 3, F and G). Thus, Ces1d expression is under direct $\mathrm{NF}-\kappa \mathrm{B}-$ dependent transcriptional control in CRC cells, both under basal and metabolic stress conditions.

Since Ces1d/CES1 specifically hydrolyses TAGs and CEs (40), we investigated whether it mediated any of the metabolic effects of NF- $\kappa \mathrm{B}$ in CRC cells. Upon Ces1d depletion, CT-26 cells exhibited a marked accumulation of TAGs and CEs, but not PCs, both at baseline and under GL (Figure 4, A-C, Supplemental Figure 4A, and Supplemental Table 1), mimicking the effects of RelA deficiency on lipid metabolism. However, Ces1d knockdown appeared to impact neutral lipids more promptly and conspicuously than the silencing of RelA (see also Figure 2, A and C), suggesting that other genes could mitigate the metabolic effects of RelA deficiency. In pulse-chase labelling experiments using $\left[{ }^{13} \mathrm{C}\right]$-oleate, TAG turnover was markedly reduced in Ces1ddeficient compared with control CT-26 cells (Figure 4D and Supplemental Table 1), and again this effect was more pronounced than that seen in RelA-depleted cells (see Figure 2E). Thus, we investigated whether Ces1d/CES1 could contribute to NF-кBdependent metabolic adaptation to energy stress conditions. As shown in Figure 4E, the silencing of Ces1d markedly diminished CT-26 cell survival following GL. Similar results were obtained using 2 alternative Ces1d-targeting hairpins (Ces1d \#2 and Ces1d \#3; Supplemental Figure 4, B and C), the structurally distinct Ces1d/CES1 inhibitors, GR-148672X (42) and WWL113 (ref. 41, Figure 4F, and Supplemental Figure 4, D and E), or MEFs (Supplemental Figure 4F). Reciprocally, ectopic Ces1d expression effectively rescued RelA-deficient cells from GL-induced death (Figure $4 \mathrm{G}$ and Supplemental Figure $4 \mathrm{G}$ ), mimicking the protective effect of oleate supplementation (Figure 2F). Moreover, a silencing-resistant but not a wild-type Ces1d-encoding lentivirus reversed GL-induced death in Ces1d-depleted CT-26 cells, thus excluding any off-target effects of the Ces1d-targeting hairpins (Supplemental Figure 4, H and I). Thus, Ces1d/CES1 mediates many of the metabolic effects of RelA on lipid catabolism and is both required and sufficient to compensate for RelA loss in controlling the response of CRC cells to starvation.

CES1 depletion impairs metabolic adaptation by reducing oxidative energy metabolism and enhancing toxic TAG accumulation. Given the essential role of Ces1d/CES1 in the NF-кB-dependent regulation of lipid catabolism and CRC cell survival during starvation, we sought to characterize the bioenergetic profile of Ces1ddeficient cells. As with etomoxir treatment, Ces1d knockdown ablated SRC in CT-26 cells, while oleate supplementation completely reversed this effect (Figure $4 \mathrm{H}$ and Supplemental Figure $4 \mathrm{~J}$ ), in keeping with our findings in RelA-deficient CRC cells (Figure 2G). Ces1d depletion also diminished the mitochondrial ATP production rate (Figure 4I and Supplemental Figure 4K). Surprisingly, however, oleate supplementation failed to rescue this impaired mitochondrial ATP production in Ces1d-depleted cells (Figure 4I and Supplemental Figure 4K). Since marked TAG accumulation was shown to induce ROS formation and mitochondrial damage, leading to cell death (43), we considered whether these events could contribute to the mitochondrial dysfunction observed in Ces1d-deficient cells during GL. Indeed, Ces1d inhibition increased both nonmitochondrial respiration and ROS production in CT-26 cells, with a corresponding depletion of cellular reduced glutathione (GSH) levels (Figure 4, J-L and Supplemental Figure 4J; see also Supplemental Figure 4, L and M). Comparable results were obtained using spectrophotometric methods to assess ROS and GSH levels (Supplemental Figure 4, N and O). The increase in ROS formation induced by Ces1d depletion was associated with enhanced lipid peroxidation, which results in ferroptosis, assessed by using BODIPY 581/591 C11 and examining 4-hydroxynonenal (HNE) protein adducts, a major end-product of polyunsaturated fatty acid (PUFA) peroxidation (Figure 4M, Supplemental Figure 4, P and Q) (44-47). This increased lipid peroxidation in Ces1d-deficient cells was accompanied by a depletion of glutathione peroxidase $4(\mathrm{Gpx} 4)$ levels, another hallmark of ferroptosis (Supplemental Figure 4R) $(46,48)$. Interestingly, enhanced ROS formation further correlated with a significant decrease of the mitochondrial membrane potential $(\Delta \Psi \mathrm{m})$ and a corresponding increase of the number of apoptotic cells upon Ces1d depletion, as shown by annexin V staining, TUNEL assays, and the assessment of caspase-3/7 activity and nucleosomal DNA fragmentation (Figure 4, N-P, Supplemental Figure 4, S-W) (44). These data suggest that ROS accumulation in Ces1d-deficient cells results in the induction of both the ferroptosis and apoptosis pathways of cell death during GL.

We reasoned that the cytotoxic effects of exaggerated TAG accumulation could explain the failure of oleate supplementation to reverse the reduced mitochondrial ATP production rate seen in Ces1d-deficient cells (Figure 4I). Consistent with this idea, the effect of Ces1d depletion on the survival of starved CRC cells was only partly recapitulated by treatment with etomoxir (Supplemental Figure 4X). Moreover, oleate supplementation failed to protect Ces1d-deficient cells from GL-induced toxicity (Figure 4Q). In contrast, the addition of Ferrostatin-1 (FR-1) and z-VAD-fmk, which block ferroptosis and apoptosis, respectively, together with oleate, completely rescued Ces1d-depleted CRC cells from GL-induced death (Figure 4Q; see also Supplemental Figure 4X). Instead, FR-1 or z-VAD-fmk either alone or in combination, but without oleate, could only partly reverse GL-induced toxicity in Ces1d-deficient cells (Supplemental Figure 4X). Collectively, these results suggest that the NF- $\mathrm{BB}$-dependent upregulation of Ces1d/CES1 mediates 2 important cytoprotective func- 
A

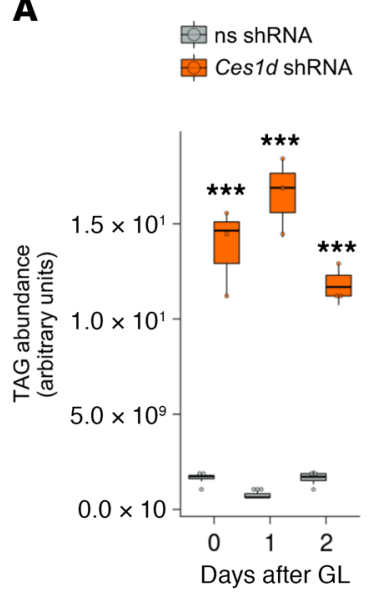

E
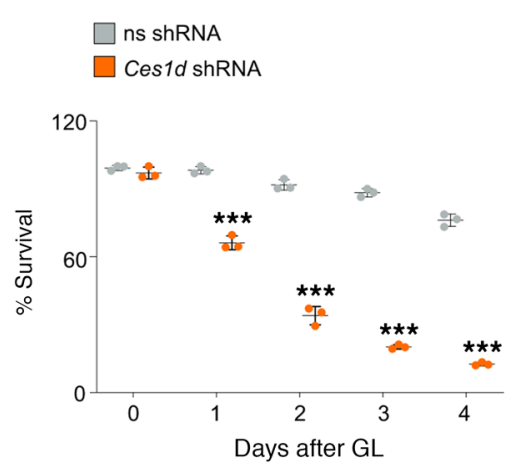

B

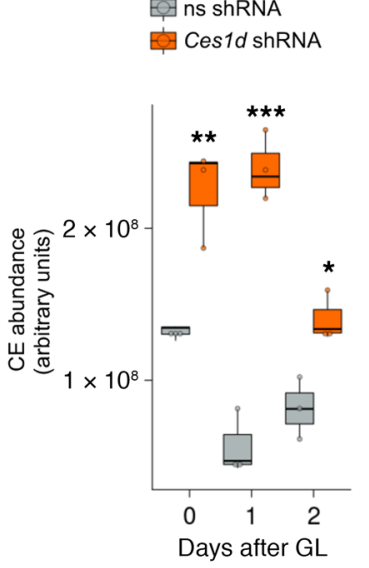

C

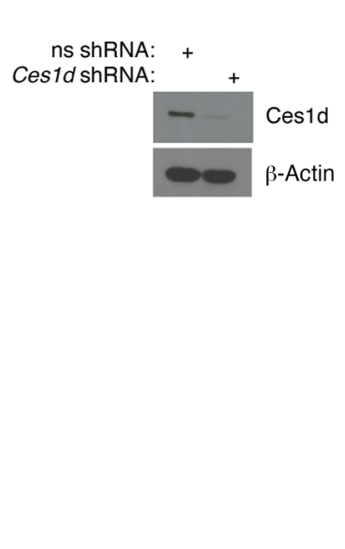

D

ns shRNA

Ces1d ShRNA

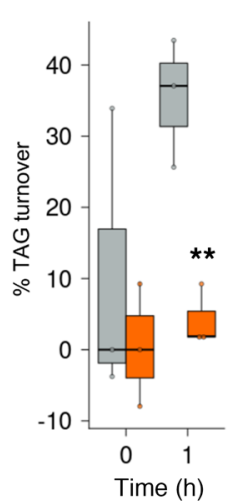

$\mathbf{F}$
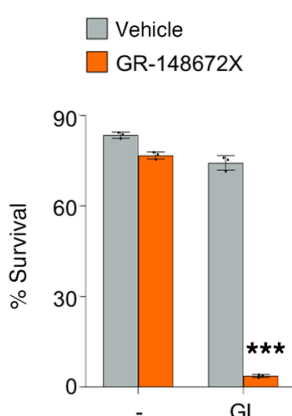

$\mathrm{GL}$
G

$\square$ ns shRNA + eGFP

$\square$ ns shRNA + Ces1d

$\square$ RelA shRNA + eGFP

$\square$ RelA shRNA + Ces1d

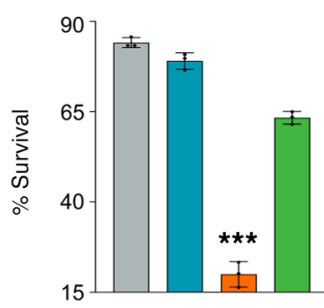

H
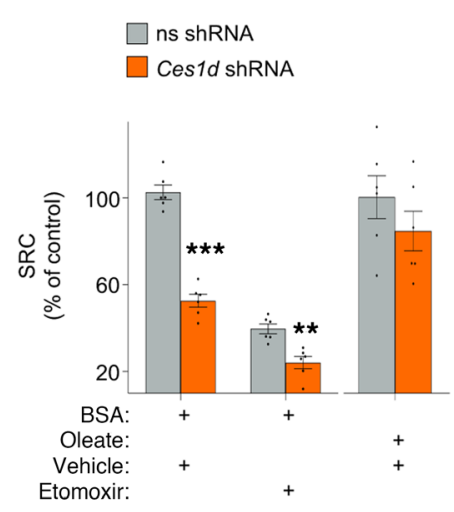

$\mathbf{L}$

M

I

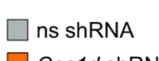

$\square$ Ces1d shRNA

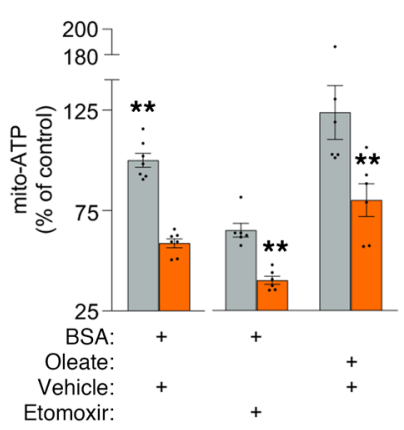

0

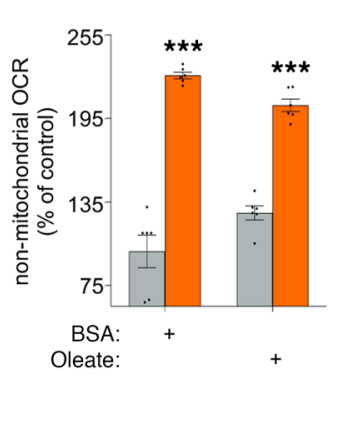

$\mathbf{K}$

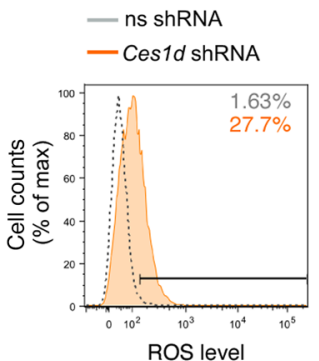

ROS level

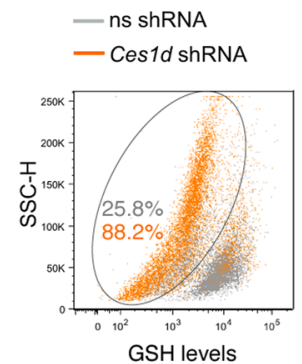

GSH levels

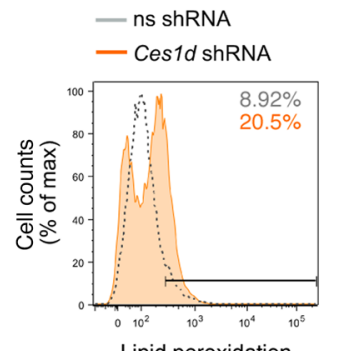

Lipid peroxidation

\section{$\mathbf{N}$}

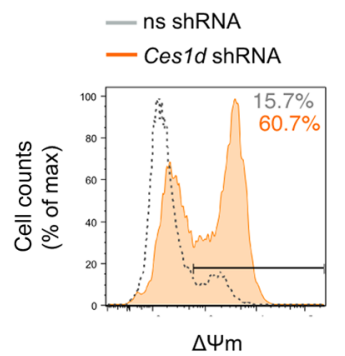

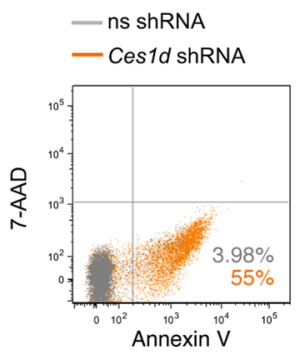

$\mathbf{P}$

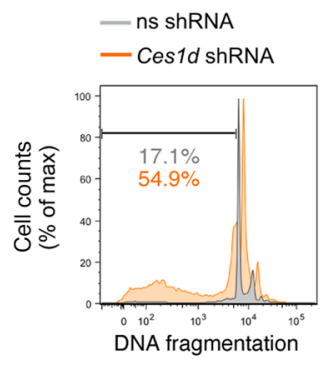

\section{Q}

$\square$ ns shRNA

$\square$ Ces1d shRNA

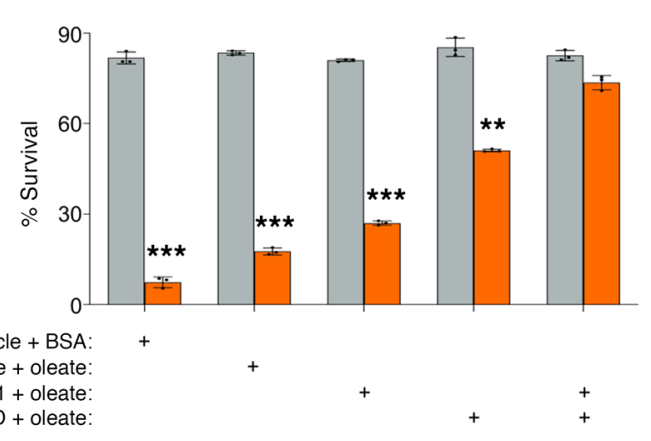


Figure 4. Ces1d mediates NF-KB-dependent metabolic adaptation by enhancing FFA catabolism and preventing TAG accumulation. ( $A$ and $B$ ) Relative TAC $(\mathbf{A} ; n=92)$ and $C E(B ; n=9)$ abundance in $C T-26$ cells expressing Ces1d or ns shRNAs at baseline (0) or under GL. (C) Western blots with cells from A. (D) TAC turnover, reporting the percentage of decrease of $\left[{ }^{13} \mathrm{C}\right]$-labeled TAG abundance $(n=40)$ at 1 versus 0 hours, in cells from $A$. ( $E$ and $\mathbf{F}$ ) Trypan blue exclusion showing the percentage of live CT-26 cells from $\mathbf{A}(\mathbf{E})$ or after a 4-day treatment as indicated (F) under normal (-) or GL conditions. (G) Trypan blue exclusion showing the percentage of live RelA-deficient or control CT-26 cells expressing eGFP or Ces1d after 4 days under GL. (H-J) SRC (H), mito-ATP (I), and nonmitochondrial OCR (J) in cells from $\mathbf{A}$ treated as shown. (K-0) FACS showing ROS levels (K), GSH levels (L), lipid peroxidation $(\mathbf{M})$, loss of mitochondrial potential $(\Delta \Psi \mathrm{m})(\mathbf{N})$, and annexin V/7-AAD positivity (0) in cells from $\mathbf{A}$ after $2(\mathbf{0}), 3(\mathbf{K}$ and $\mathbf{N})$ or 4 ( $\mathbf{L}$ and $\mathbf{M}$ ) days under $\mathrm{GL}$. (P) Propidium iodide staining showing apoptotic DNA fragmentation in cells from $\mathbf{A}$ after 4 days under $\mathrm{CL}$. (Q) Trypan blue exclusion showing the percentage of live cells from $\mathbf{A}$ treated as indicated after 4 days under GL. (A, B, and D) Shown are the medians (horizontal lines), 25th to 75th percentiles (box outlines), and highest and lowest values within 1.5 times the interquartile range (vertical lines). (E, F, and $\mathbf{Q}$ ) Values denote means $\pm S D(n=3)$. $(\mathbf{H}-\mathbf{J})$ Values denote means \pm SEM ( $n$ $=6)$. (A, B, D-J, and Q) Significance was calculated by 2-tailed Student's $t$ test. ${ }^{*} P<0.05 ;{ }^{* *} P<0.01 ;{ }^{* * *} P<0.001$.

tions in CRC cells during metabolic stress: first, to maintain ATP homeostasis and thus enable cells to meet their energy demand by increasing the FFA supply to fuel FAO and OXPHOS; second, to prevent the toxic build-up of neutral lipids that results in ROS production and mitochondrial damage, triggering apoptosis and ferroptosis (43).

CES1 expression portends worse clinical outcomes in overweight patients with CRC. The maintenance of energy homeostasis is essential for malignant cell survival in the nutrient-insufficient TME (1-4). We therefore investigated whether CES1dependent TAG catabolism could contribute to the pathogenesis of CRC, since FAO is also involved in stem cell renewal, anchorage independent growth, and metastatic spread (15-17). Fifty-nine patients with CRC were stratified on the basis of tumor-associated CES1 expression and the lipid composition of their tumors was analyzed by MS. These analyses showed that elevated CES1 expression correlated with a reduced abundance of multiple TAG species in the tumor tissue, compared with tumors exhibiting low CES1 mRNA expression (Figure 5A, Supplemental Figure 5, A and B, Supplemental Table 1), consistent with our findings in CRC cell lines (Figure 4A). In the same CRC patient cohort, elevated CES1 expression also correlated with shorter disease-specific survival (DSS) (Figure 5B), and interestingly, this correlation demonstrated an increased significance in overweight patients (Figure 5C). Notably, we found no prognostic correlation with CES1 expression in the nonoverweight CRC patient subgroup (Figure 5C). Similar results, demonstrating a significant association between elevated CES1 expression and both shorter progression-free interval (PFI) and shorter DSS in overweight, but not nonoverweight patients, were obtained upon analysis of an external CRC data set from The Cancer Genome Atlas (TCGA) network using 25th percentile as cut-off value for CES1 expression (ref. 49, Figure 5, D and E, and Supplemental Figure 5, C and D). Together, these results identify CES1 as a hallmark of aggressive disease in overweight patients with CRC.
Since obesity is a major risk factor for CRC and increases both the disease recurrence and mortality rates in patients with CRC (50-52), we sought to investigate in more detail its association with CES1 expression. Interestingly, the analysis of CRC data sets demonstrated that the obesity gene signature and percentage of overweight patients were both significantly increased in the mesenchymal CMS4 subtype compared with other CRC subtypes (Figure 5, F and G and Supplemental Figure 5, E and F). Notably, CES1 expression was correspondingly upregulated in the aggressive CMS4 subtype, in keeping with our findings of a correlation between CES1 expression and worse prognosis in CRC (Figure $5 \mathrm{H}$ and Supplemental Figure 5G). Of note, CES1 expression was also upregulated in the canonical CMS2 CRC subtype (discussed below). Thus, our analyses of human data sets (33) demonstrated a clear stratification of CES1 expression with clinically relevant core molecular subtypes of CRC, obesity-related inflammation, and patient outcome. Notably, no other intracellular TAG lipase displayed such a distinctive CRC subtype-based distribution, nor a correlation with reduced survival in patients with CRC (Supplemental Figure 5, H-R). Together, these findings suggest a unique role for CES1 in the pathogenesis of aggressive CRC.

Elevated CES1 expression is driven by NF- $\kappa B$ activation in CMS4 CRC and by HNF4A amplifications in CMS2 CRC. The high CES1 expression observed in CMS4 CRCs was consistent with the elevated NF-кB-activation and inflammatory gene signatures characteristic of these tumors. However, the increased CES1 expression seen in the CMS2 subtype was unexpected, since this subtype is not linked to inflammation nor NF- $\mathrm{KB}$ activation (Figure $5 \mathrm{H}$ ). We considered that CMS2 CRCs are enriched in amplifications of the gene encoding the transcription factor, hepatocyte nuclear factor $4 \alpha(\operatorname{HNF} 4 \alpha)$ (33), residing in the chromosome 20q11-q13 amplicon, which correlates with worse clinical outcomes in patients with CRC $(49,53)$. Since HNF $4 \alpha$ is a transcriptional regulator of CES1 (54), this raised the possibility that increased CES1 expression in CMS2 CRCs could be driven by $H N F 4 A$ amplifications. Consistent with this idea, $H N F 4 A$ was expressed at significantly higher levels in the CMS2 than other CRC subtypes (Figure 5I and Supplemental Figure 5S). Moreover, CES1 expression correlated with the presence of $H N F 4 A$ amplifications, as well as HNF4A mRNA expression in CRC data sets (Supplemental Figure 5, T and U). Interestingly, CES1 expression in human CRCs also correlated with the NF- $\kappa \mathrm{B}$ target-gene signature (Supplemental Figure 5V), which is upregulated in the CMS4 subtype along with CES1 (Figure $1 \mathrm{~A}$ and Figure $5 \mathrm{H}$ ).

These analyses of patients with CRC suggested that elevated CES1 expression is largely driven by $\mathrm{NF}-\kappa \mathrm{B}$ activation in the CMS4 subtype and by HNF4A amplifications in the CMS2 subtype, underscoring the importance of CES1 upregulation in the etiopathogenesis of CRC. Consistent with this idea, NF- $\mathrm{kB} / \mathrm{RelA}$ was activated by GL-induced Ser536 phosphorylation in 3 of the 6 human CRC cell lines evaluated (Supplemental Figure 6A). Accordingly, RelA knockdown decreased CES1 expression, resulting in TAG and CE but not PC accumulation and impaired metabolic adaptation during energy stress only in the 3 CRC cell lines in which NF- $\mathrm{BB}$ was activated by GL (Figure 6, A-C and Supplemental Figure 6, A-C). Strikingly, despite this cell-selective NF- $\mathrm{B}$ activation, all $6 \mathrm{CRC}$ cell lines depended on CES1 expression for 
A

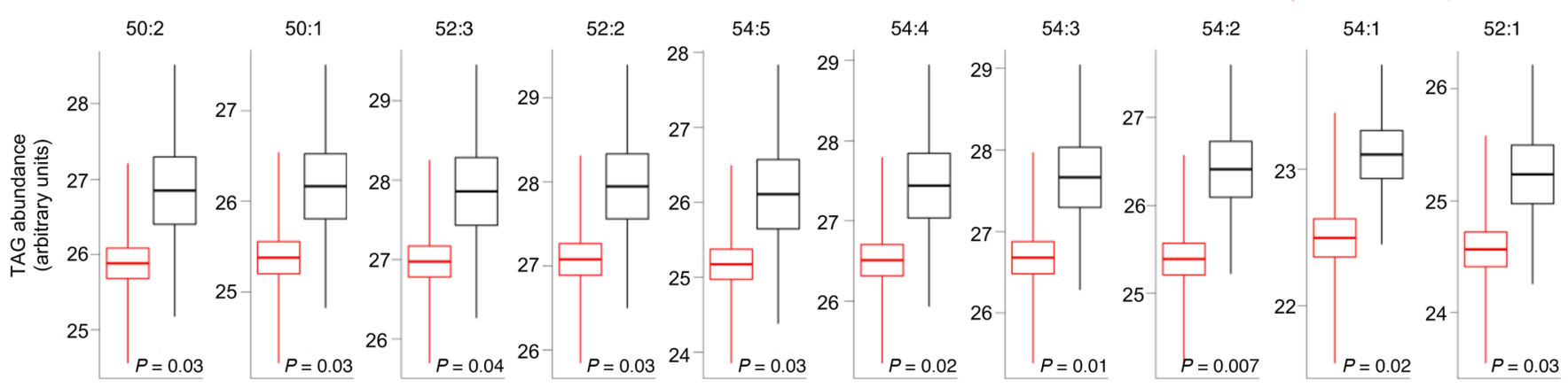

B

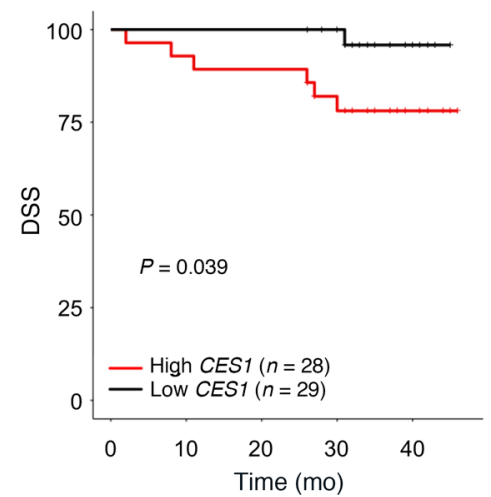

D

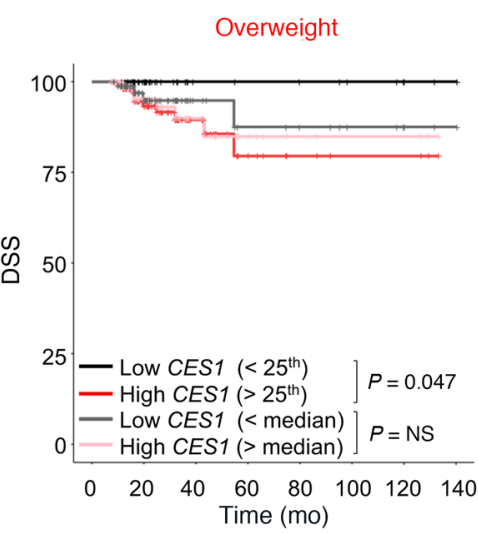

E

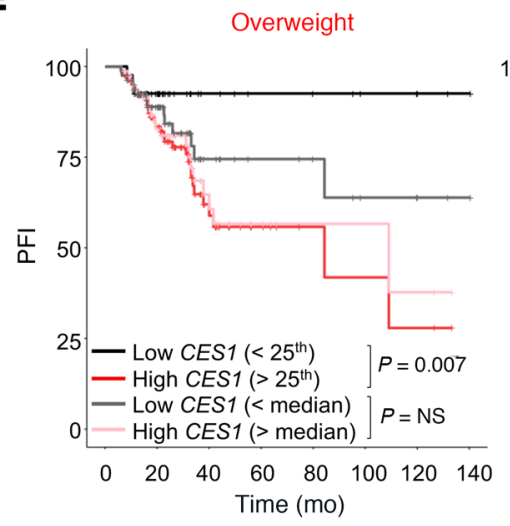

C

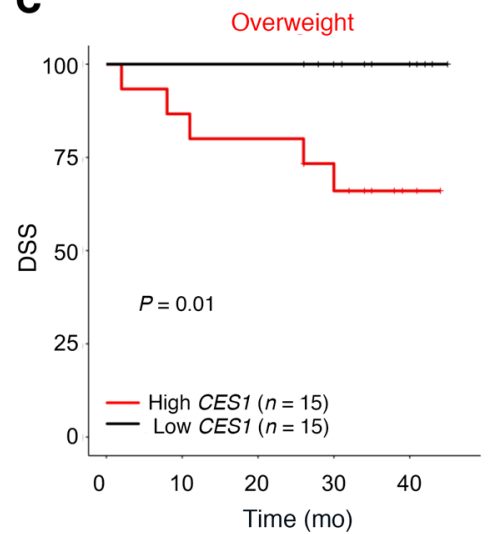

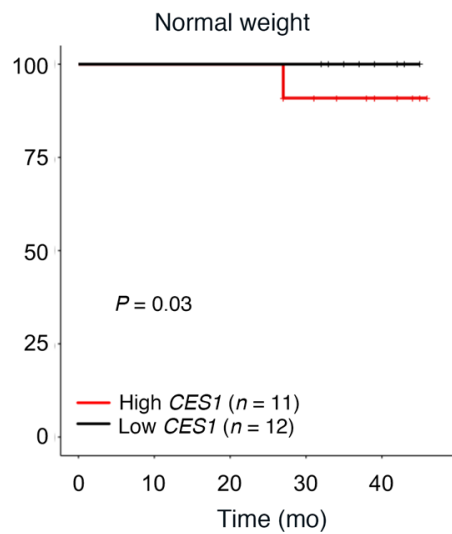
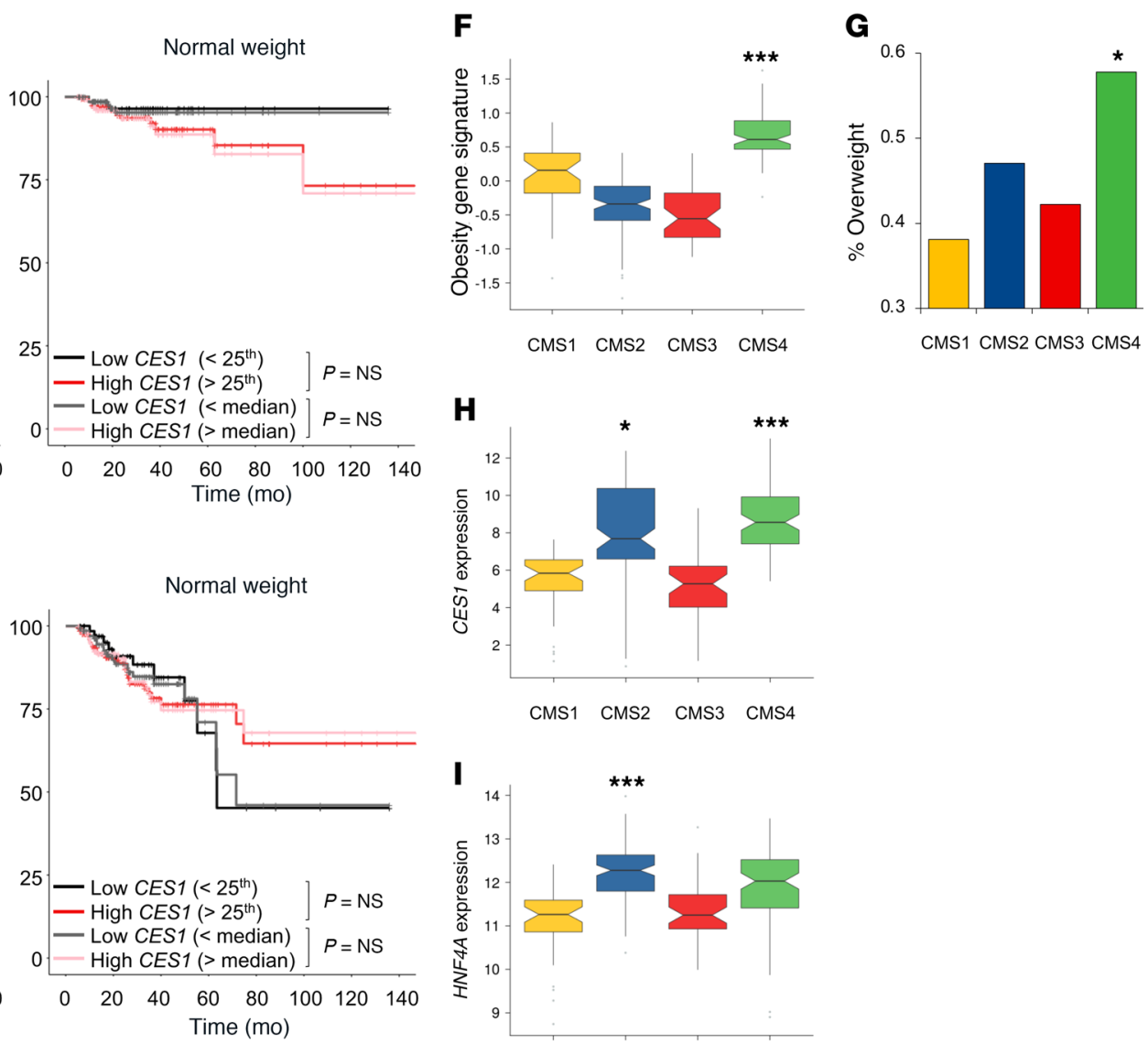

Normal weight

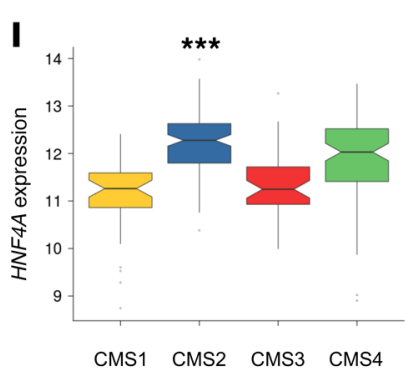


Figure 5. CES1 is upregulated in CMS2 and CMS4 CRCs and denotes aggressive disease in obese/overweight patients. (A) TAG abundance in CRCs $(n=57)$ stratified based on CES1 expression. Shown are the mean (horizontal lines), mean \pm SEM (box outlines), or mean \pm SD (vertical lines). Significance was calculated by 2-tailed Student's $t$ test. (B) DSS in patients from A stratified based on tumor-associated CES1 expression. (C) DSS in obese/overweight (left; $n=30$ ) and normal weight (right; $n$ = 23) patients from $\mathbf{A}$ stratified as in B. (D) DSS in obese/overweight (left; $n=157$ ) and normal weight (right; $n=272$ ) patients with CRC from the TCGA data set $(n=427)$ stratified based on tumor-associated CES1 expression using the 25 th or 50 th percentile as thresholds. (E) PFI in obese/overweight (left; $n=161$ ) and normal weight (right; $n=275$ ) patients from $\mathbf{D}$ stratified as in $\mathbf{D}$. (F) Median $z$ scores of the obesity-gene signatures in each CMS CRC subtype from Figure 1, A and B. (G) Percentage of overweight patients with CMS subtypes from F. Samples in each subtype were compared with all other CRC samples using Fisher's exact test. ${ }^{*} P=0.03$. (H and $\left.\mathbf{I}\right) C E S 1$ (H) and HNF4A (I) mRNA expression in CMS subtypes from $\mathbf{F}$. (B-E) Significance was calculated using the log-rank test. (F, H, and I) Shown are the medians (horizontal lines), 25th to 75 th percentiles (box outlines), and highest and lowest values within 1.5 times the interquartile range (vertical lines). Notches denote the $95 \%$ confidence interval of the medians. Samples in each CMS subtype were compared with all other CRC samples using 2-tailed Student's $t$ test. Significance for multiple comparisons was calculated using the KruskalWallis test $\left(P<2.2 \times 10^{-16}\right)$. ${ }^{*} P<0.05 ;{ }^{* *} P<0.001$.

survival during GL, as shown by the marked toxicity produced by CES1 deficiency in starved CRC cells (Figure 6C). Similar results were obtained using 2 independent CES1-targeting hairpins (CES1 \#2 and CES1 \#3; Supplemental Figure 6, D and E). These data suggested that CES1 is the dominant effector of NF- $\mathrm{\kappa B}$-dependent metabolic homeostasis in CRC cells during GL, because RelA knockdown had no effect on CRC cell survival under energy stress conditions, unless it resulted in a decrease of CES1 expression (Figure 6A and Supplemental Figure 6C). In contrast, HNF4A expression was higher in the RelA-independent than RelA-dependent CRC cell lines (Supplemental Figure 6F) and, correspondingly, HNF4A knockdown diminished constitutive CES1 expression in the 3 RelA-independent but not the RelA-dependent CRC cell lines (Figure 6D and Supplemental Figure 6G). Two alternative HNFA4-targeting hairpins produced similar effects (Supplemental Figure 6G). Collectively, our results support the hypothesis that CES1 transcription is reciprocally controlled by NF- $\mathrm{BB}$ and HNF $4 \alpha$ in distinct subsets of CRC.

CES1 is a potentially actionable therapeutic target in aggressive CRC. Given the profound effect of CES1 depletion on CRC cell survival upon GL and the clinical relevance of CES1 expression in CRC pathogenesis, we hypothesized that blockade of the CES1 catalytic activity could provide an effective therapeutic strategy to treat patients with aggressive forms of CRC. In support of this hypothesis, treatment with the CES1 inhibitor, GR148672X (42), significantly increased GL-induced cell death in all human CRC cell lines investigated, while demonstrating no overt toxicity under normal culture conditions (Figure 6E and Supplemental Figure $6 \mathrm{H})$. Similar results were obtained using a second CES1 inhibitor (WWL113; Supplemental Figure 6, I and J) (41). These results indicated that the effects of CES1 knockdown on CRC cell survival after GL were due to reduced CES1 enzymatic activity rather than impaired CES1 adaptor function. These findings also confirmed the contextual specificity of the CES1- targeting approach for metabolic stress conditions. Interestingly, in a mouse allograft model of CRC, treatment with GR148672X over a 10-day period significantly reduced tumor growth, with no apparent adverse effects (Figure 7, A and B and Supplemental Figure 7A). At the endpoint, all control mice had developed large tumors, whereas mice treated with GR148672X displayed a significant reduction of both tumor volume and weight (Figure 7, B-D). Similar results showing a marked drug-dependent inhibition of CRC oncogenesis with no apparent toxicities were obtained with GR148672X in a second mouse xenograft model of CRC, using the human cell line HCT-116 (Figure 7, E-H and Supplemental Figure 7B). These results are broadly consistent with a previous study, which reported a significantly reduced growth of B cell tumors in Ces1d-deficient mice (55). Collectively, these data identify CES1 as a potentially actionable therapeutic target in aggressive core CRC subtypes and support the therapeutic potential of blocking CES1 to counter colorectal carcinogenesis.

\section{Discussion}

Our findings uncover a metabolic mechanism mediated by CES1 that drives CRC pathogenesis downstream of inflammatory $\mathrm{NF}-\mathrm{\kappa B}$ signaling and HNF4A amplification in the CMS4 and CMS2 subtypes, respectively. CES1 enhances CRC cell survival under energy stress conditions by cell-autonomously increasing FFA availability for ATP production and preventing TAG-induced cytotoxicity mediated by ROS and phospholipid peroxidation (Figure 7I). Our results also provide a mechanism for the role of NF- $\mathrm{kB}$ in the etiopathogenesis of CRC, revealing an intriguing association that links NF- $\mathrm{KB}$ activity and inflammation with obesity and fat metabolism in the mesenchymal CMS4 subtype. Further, our data provide a basis for the pathogenic role of prognostically unfavorable HNF4A gene amplifications in patients with CRC (53). Thus, despite using entirely different genetic and inflammatory oncogenic pathways, respectively, CMS2 and CMS4 tumors both rely on the same CES1-mediated downstream mechanism to withstand starvation and drive malignant CRC evolution. Future studies will clarify the precise mechanisms for the CES1 transcriptional regulation by NF- $\mathrm{KB}$ and HNF4 $\alpha$ in human CRC. Notwithstanding, our results underscore the central metabolic role of CES1 in CRC etiopathogenesis.

Strong epidemiological evidence demonstrates that the recurrence and mortality rates of patients with CRC markedly increase in obese people, underscoring the importance of lipid metabolism in CRC pathogenesis (50-52). A prominent feature of CRC in obese patients is a chronic state of low-grade inflammation that is exacerbated by the anatomical proximity of tumors to abdominal hypertrophic adipose depots, resulting in alterations of cancer cell metabolism and biology, which are thought to promote CRC progression $(51,52,56)$. Yet surprisingly little is known about how altered lipid metabolism contributes to the aggressive evolution of CRC in obese patients. We find that, despite the remarkable genetic and phenotypic heterogeneity of CRC (57), CES1 expression markedly increases in the metastasis-prone mesenchymal CMS4 subtype and portends worse clinical outcomes in overweight/obese patients. It was previously suggested that cancer cells use fatty acids released by TME-based adipocytes and stromal cells to support rapid tumor growth $(17,58)$. Our data do not 
A

$\square$ ns ShRNA

$\square$ RELA ShRNA

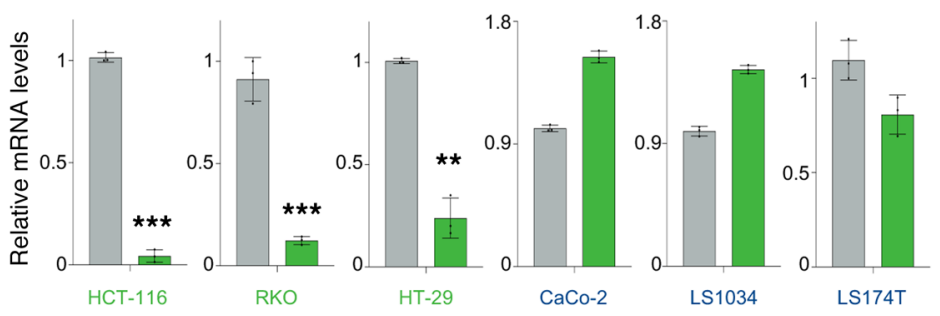

C

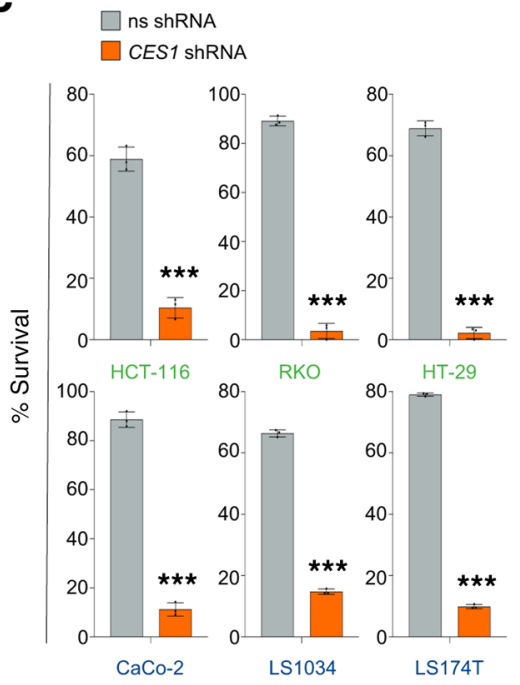

ns ShRNA:

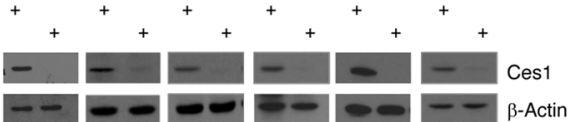

HCT-116 RKO HT-29 CaCo-2 LS1034 LS174T

$\square$ ns shRNA
B

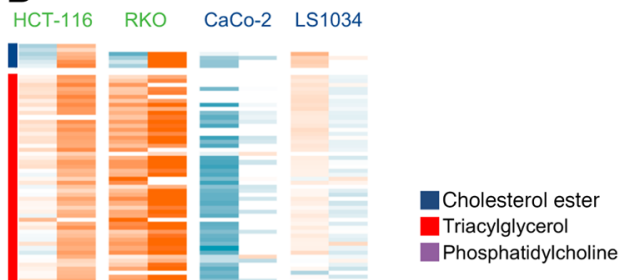

$\mathbf{E}$
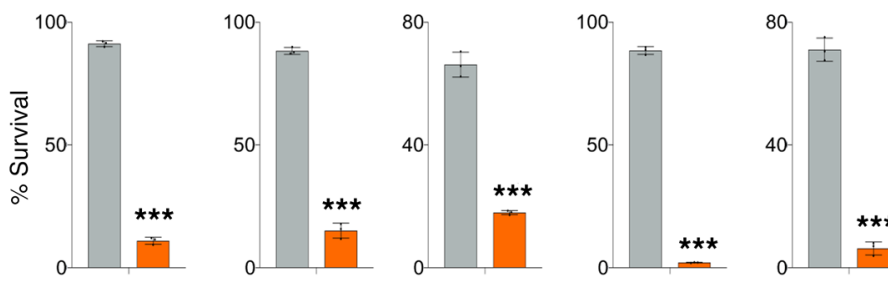

100

HNF4A ShRNA
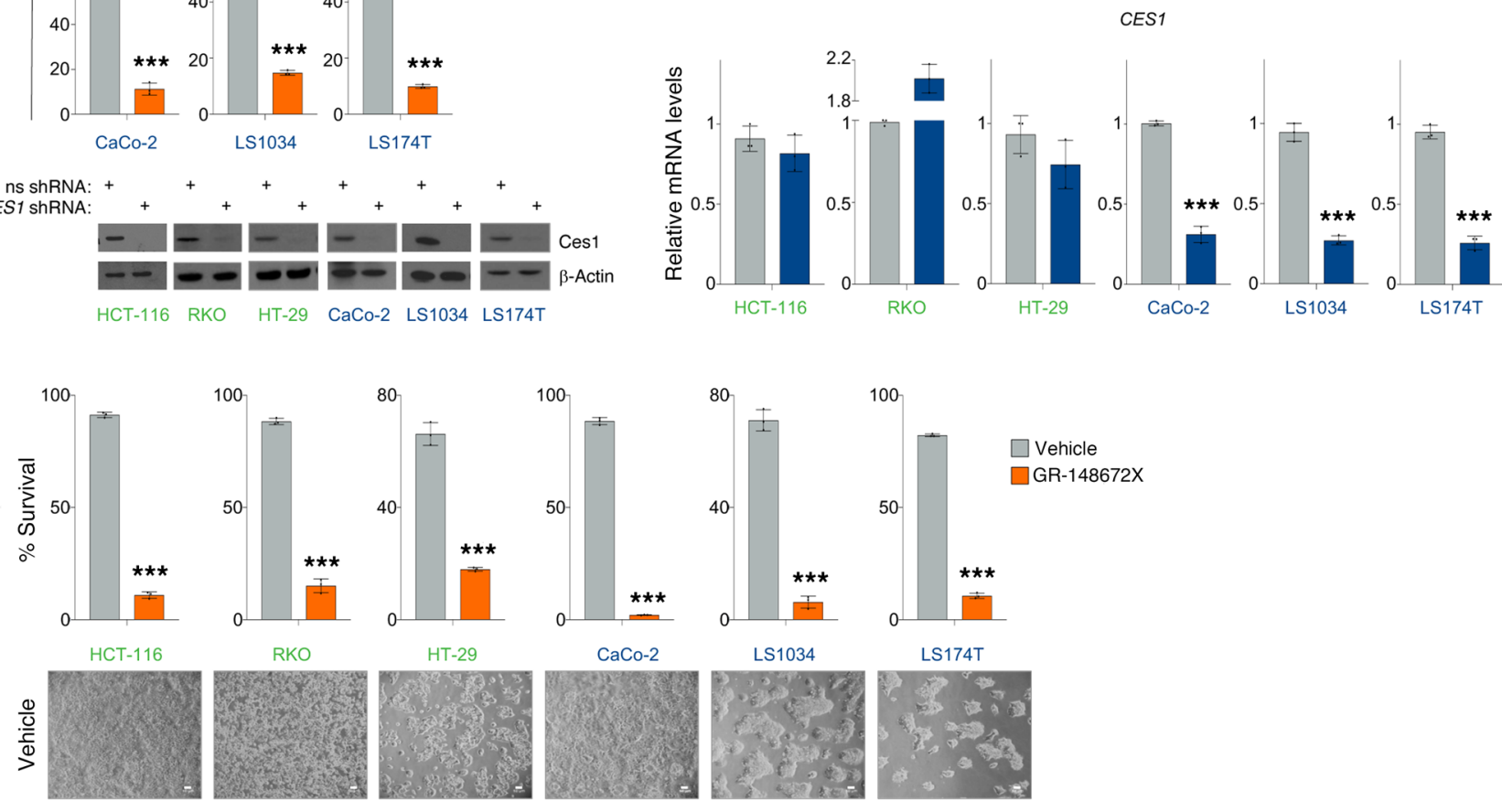

$\square$ Vehicle

$\square$ GR-148672X
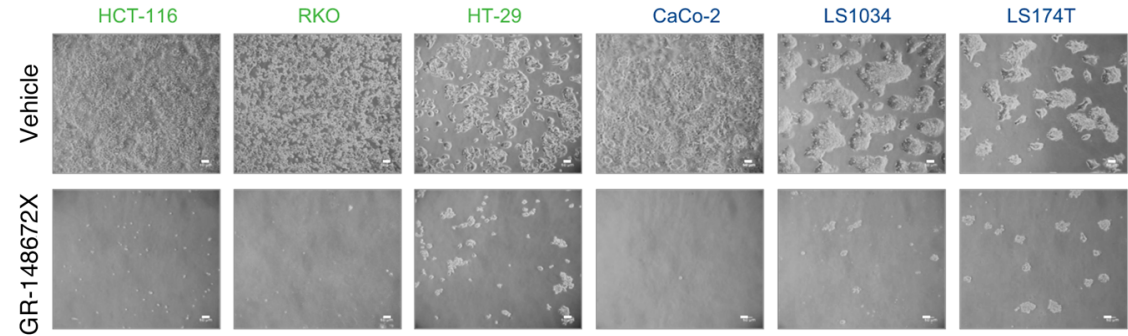
Figure 6. CES1-dependent metabolic adaptation is reciprocally controlled by NF-KB and HNF4 $\alpha$ in distinct CRCs. (A) qRT-PCR showing CES1 mRNA expression in the indicated human CRC cell lines expressing RELA-specific (RELA) or ns shRNAs. (B) Heatmap showing the $\log _{2}$ fold change of metabolic species in the indicated lipid classes in RELA-deficient (RELA) relative to control cells in the indicated human CRC cell lines, cultured under normal conditions ( 0 ) or GL for 4 days, identified by LC-MS and Lipostar software. Shown are the lipids having a value different from zero at the time points investigated. Orange, increased abundance; azure, decreased abundance. Statistical significance of the lipid accumulation during $\mathrm{GL}$ was determined by a hypergeometric test, corrected using the Benjamini-Hochberg procedure (triacylglycerols in RKO cells at $\mathrm{d} 4, q=5.47 \times 10^{-32}$ ). (C) Trypan blue exclusion assays showing the percentage of live cells from the indicated human CRC cell lines expressing CES1-specific (CES1) or ns shRNAs after a 4-day culture under GL (top). Western blots showing CES1 and $\beta$-actin protein levels in the same cells (bottom). (D) qRT-PCR showing the CES1 mRNA expression in the human CRC cell lines from $\mathbf{A}$ expressing HNF4A-specific (HNF4A) or ns shRNAs. (E) Trypan blue exclusion assays showing the percentage of live cells from the indicated human CRC cell lines after a 4-day treatment with GR-148672X $(10 \mu \mathrm{M})$ or vehicle during $\mathrm{CL}$ (top). Values denote mean $\pm \operatorname{SD}(n=3)$. Images of representative cells (bottom). Scale bar: $50 \mu \mathrm{m}$. (A and D) Experiments were conducted twice. (C and $\mathbf{E}$ ) Experiments were conducted at least 3 times. (A and $\mathbf{C}-\mathbf{E}$ ) Values denote mean $\pm \operatorname{SD}(n=3)$. Statistical significance was calculated by 2-tailed Student's $t$ test. ${ }^{* *} P<0.01 ;{ }^{* *} P<0.001$.

exclude a role for this metabolic symbiosis between cancerous and noncancerous cells in CRC, nor an additional role for CES1 in tumor-associated adipocytes. However, our findings from the investigations of patients with CRC and preclinical cellular and animal models underscore the importance of the cell-autonomous mechanism mediated by CES1 in mobilizing FFAs from endogenous lipid reservoirs for supporting CRC cell survival under energy stress conditions. Indeed, these endogenous FFAs would presumably represent the only source of FAO fuels available to CRC cells for maintaining metabolic homeostasis upon loss of attachment or migration outside the TME during the initial stages of metastasis (15). By increasing FFA availability and oxidative energy metabolism, elevated CES1 expression could also link inflammation with the EMT in inflamed CMS4 tumors, in keeping with the roles of FAO and inflammation in both driving and maintaining stemness in cancer $(15,18)$.

The identification of actionable therapeutic targets that are linked to the pathogenesis of core molecular tumor subtypes is an area of major interest and clinical need, particularly in obesityassociated mesenchymal tumors, given their higher risk of metastasis, relapse, and resistance to cancer therapy (57). However, translating oncological metabolic targets to the clinical setting has been complicated by the frequent toxicities produced by the suppression of mechanisms that are also required for normal cell function $(59,60)$. Interestingly, in this regard, CES1 appears to be a promising therapeutic target in CRC, given its contextual specificity for energy stress conditions and its selective overactivation in microsatellite stable/nonhypermutated CMS4 (mesenchymal) and CMS2 tumors, which are generally refractory to immune-checkpoint blockade immunotherapy. Accordingly, as well as systemic pharmacologic inhibition, genetic Ces1d deletion appears to be well tolerated in mouse models, because Cesld knockout mice are viable, lean, and seemingly healthy (61). Indeed, the short half-life and poor stability of the tool compounds used in our study underscore the therapeutic potential of the CES1-dependent metabolic mechanism in CRC and the need for developing novel CES1 inhibitors with improved bioavailability. Thus, while further investigations will determine whether CES1 blockade can be developed into an effective treatment strategy in obese patients with CRC, our findings may serve as an example for developing tumor subtype-based interventions that target core tumor cell vulnerabilities also in cancers beyond CRC.

\section{Methods}

A detailed discussion of the Methods is reported in the Supplementary Material.

Data availability. mRNAseq data set generated in the current study (Figure 3A) is included in the Supplementary Material files as Supplemental Table 2 and has been submitted to the European Nucleotide Archive under accession number PRJEB32382.

The Metabolic Profiling data sets (general platform and lipid platform) generated in the current study (Figure 1, D and E; Figure 2, A, C-E; Figure 4, A, B, and D; Figure 6B; Supplemental Figure 2A; and Supplemental Figure 4A) are included in the Supplementary Material files as Supplemental Table 1.

The lipid profiling data set generated from patients with CRC is included in the supplementary material as Supplemental Table 1.

Data from the analysis of The Cancer Genome Atlas (TCGA) CRC (COADRED) program and the French National Cartes d'identite des Tumeurs (FNCIT) program were classified according to the CMS algorithms described by the Colorectal Cancer Subtyping Consortium (CRCSC) and downloaded using the Synapse browser (ID: syn2623706). Additional information is available in the section "CRC patients and human CRC data sets" of the Supplemental Methods.

Study approval. A prospective, observational study of patients at serial stages along the CRC disease pathway was conducted between January 2015 and January 2017. Ethical approval for this study was provided by the Research Ethics Committee and Health Research Authority (REC reference: 14/EE/0024). Informed written consent was obtained from all the subjects involved in the studies. Mouse experiments were conducted under the authority of UK Home Office PPL P57F16A53. The PPL was approved by the Imperial College Ethical Review Process and the UK Home Office.

\section{Author contributions}

G Franzoso and DC conceived the project and designed the experiments. DD conceived and designed the bioinformatic analysis and performed the statistical analysis of the data. DC, FB, LT, SCL, JKE, DV, JB, and LS performed and analyzed the biological experiments. DC, LG, ADV, GC, JKE, HCK, and KB conducted the metabolic analyses and processed and analyzed the data. DD, JLA, JSM, and MLD performed the metabolic profiling of CRC patient samples and the analysis of the clinical data. SEM, ZT, and JMK contributed CRC clinical samples and metabolic and clinical analyses of patient samples. TSV, YM, WS, G Frost, and VT contributed key reagents and technical expertise. KRC performed the analysis of the Ces1d promoter. G Franzoso, DC, and DD wrote the manuscript. All authors read and edited the manuscript. DC is listed before DD as co-first author because she initiated the project. 
A

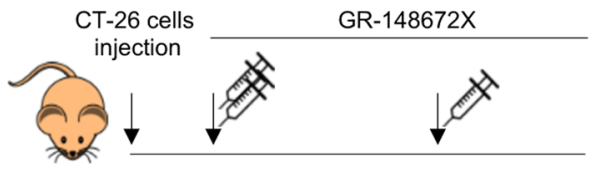

Time (d) $\quad 0 \quad 3$

9

13

B

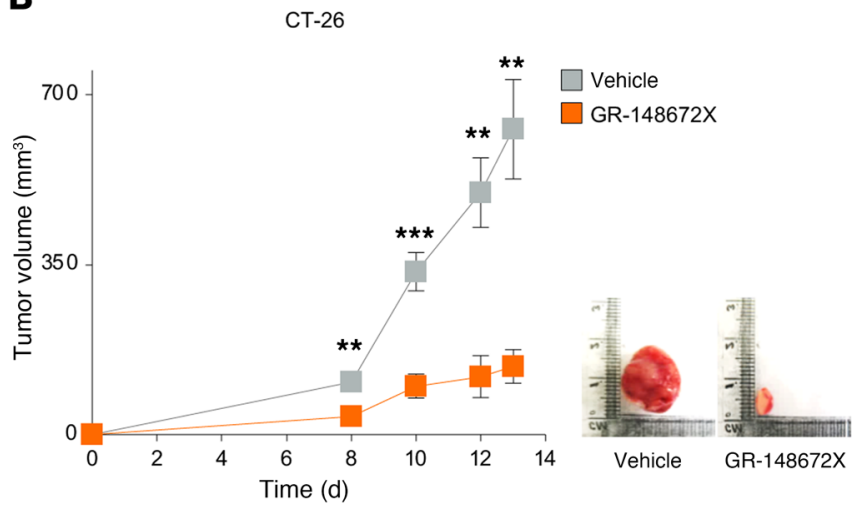

C

CT-26

D
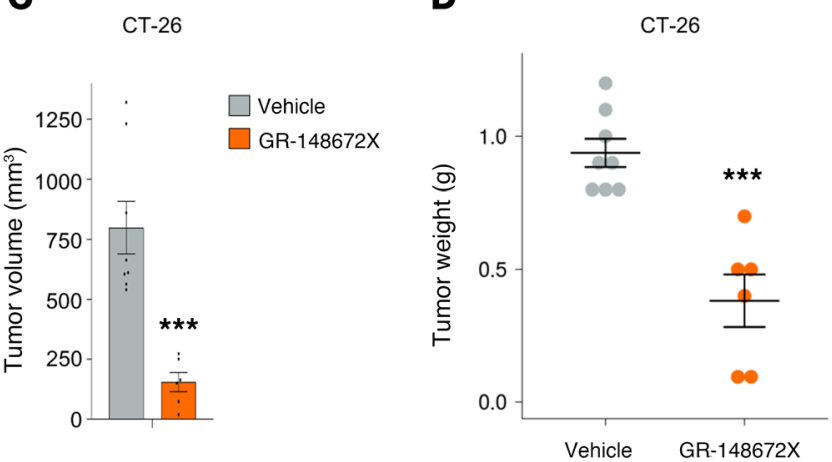

$\mathbf{E}$

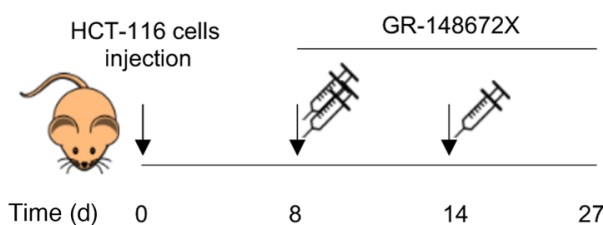

$\mathbf{F}$

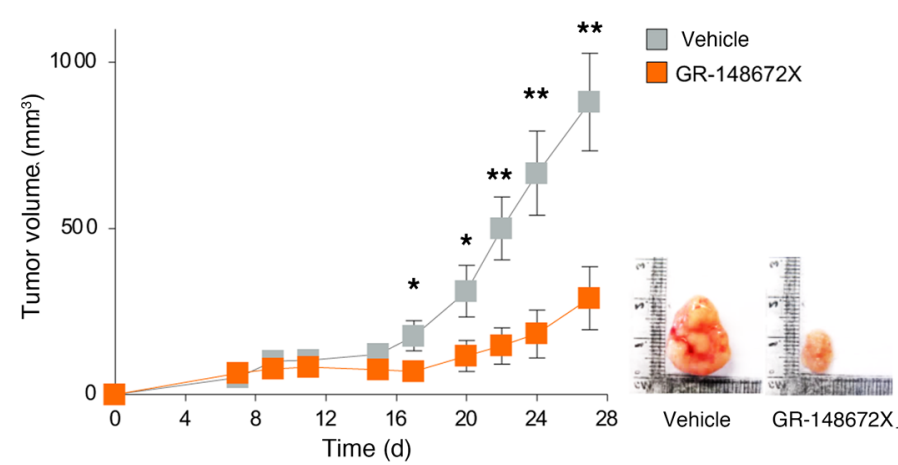

G

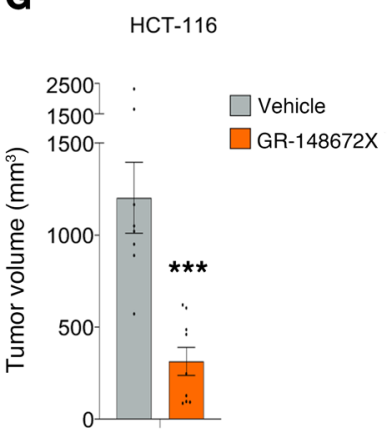

H

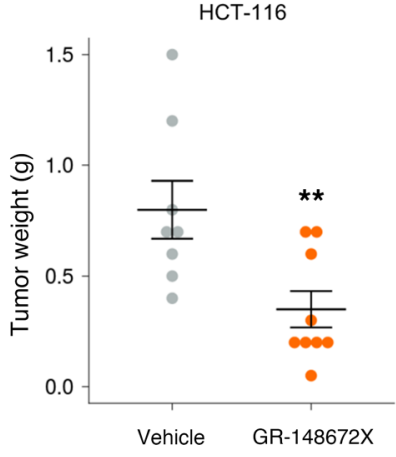

I

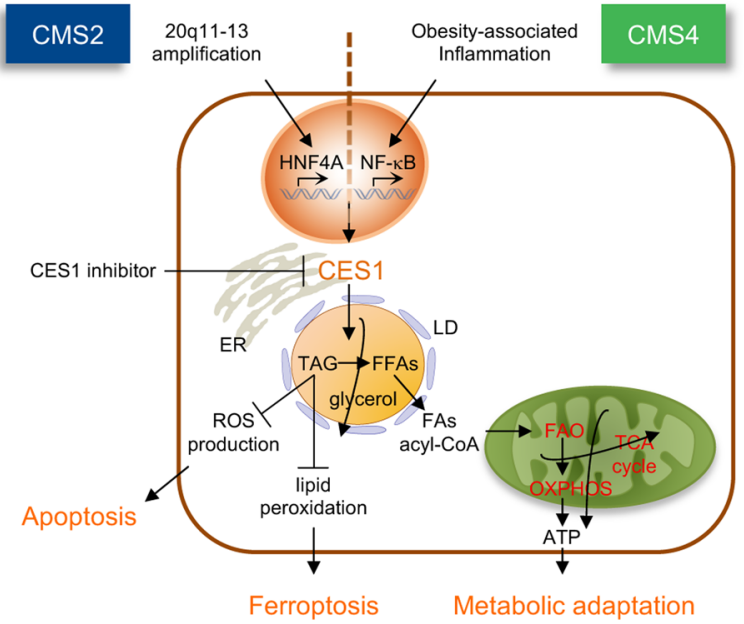

Figure 7. Pharmacologic Ces1d/CES1 inhibition counters CRC tumorigenesis in vivo. (A) Diagram summarizing the treatment schedule used in B. (B) Volumes of subcutaneous CT-26 CRC allografts in mice treated by intraperitoneal injection of GR-148672X (50 mg/ $/ \mathrm{kg}$ ) or vehicle as shown (left). Images of representative tumors isolated from mice at day 13 (right). (C) Volumes of subcutaneous CT-26 CRC tumors from B measured ex vivo at day 13. (D) Dot plots showing the weight of tumors from the mice in $\mathbf{B}$ at day 13. (E) Diagram summarizing the treatment schedule used in F. (F) Volumes of subcutaneous HCT-116 CRC xenografts in mice treated by intraperitoneal injection of GR-148672X (50 mg/ $\mathrm{kg}$ ) or vehicle as shown (left). Images of representative tumors isolated from mice at day 27 (right). (C) Volumes of subcutaneous HCT-116 CRC tumors from $\mathbf{F}$ measured ex vivo at day 27. (H) Dot plots showing the weight of tumors from the mice in $\mathbf{F}$ at day 27. (I) Schematic representation of the reciprocal regulation of CES1 expression by NF- $\mathrm{KB}$ and HNF4A in the CMS4 and CMS2 CRC subtypes, respectively. Depicted is the CES1-mediated metabolic mechanism increasing TAC breakdown to fuel FAO and OXPHOS during starvation, while preventing the toxic build-up of neutral lipids that triggers apoptosis and ferroptosis. LD, lipid droplet. (B-D and F-H) Values denote mean \pm SEM. (B-D) GR-148672X-treated mice, $n=6$; vehicle-treated mice, $n=8$. (F-H) GR-148672X-treated mice, $n=$ 9; vehicle treated mice, $n=8$. ( $(\mathbf{D}$ and $\mathbf{H})$ Horizontal lines and whiskers denote median \pm SEM. (B-D and $\mathbf{F}-\mathbf{H}$ ) Statistical significance was calculated by 2 -tailed Student's $t$ test. ${ }^{*} P<0.05 ;{ }^{* *} P<0.01 ;{ }^{* *} P<0.001$. 


\section{Acknowledgments}

We thank Steven Ley, Jacques Behmoaras, Navdeep Chandel, Richard Lehner, David Carling, and Antonio Iavarone for critical comments on the manuscript. We also thank Enrico De Smaele for the Tween lentiviral vector. This work was supported in part by Cancer Research UK program grant A15115, Medical Research Council (MRC) Biomedical Catalyst grant MR/L005069/1, Bloodwise project grant 15003, and NIHR Imperial Biomedical Research Centre (BRC) ITMAT Push for Impact Award P81135 to G Franzoso. Infrastructure support for this research was provided in part by the NIHR Imperial BRC. LG, ADV, and GC acknowledge the Università degli Studi di Perugia and MIUR for financial support to the project AMIS, through the program "Dipartimenti di Eccellenza 2018-2022". VT and TSV were supported by National Research Foundation award NRF-CRP17-2017-02.
Address correspondence to: Guido Franzoso or Daria Capece, Imperial College London, CWB, Du Cane Road, W12 ONN, London, United Kingdom. Phone: 44.020.3313.8421; Email: g.franzoso@imperial.ac.uk (GF). Phone: 44.020.8383.8430; Email: d.capece@imperial.ac.uk (DC).

DD's present address is: MRC Centre for Neuropsychiatric Genetics and Genomics, Cardiff University, Cardiff, United Kingdom.

SCL's present address is: Agency for Science, Technology and Research (A*STAR), Singapore.

WS's present address is: Rhea Pharmaceutical Sciences Pte Ltd, Singapore.
1. Cairns RA, et al. Regulation of cancer cell metabolism. Nat Rev Cancer. 2011;11(2):85-95.

2. Pavlova NN, Thompson CB. The emerging hallmarks of cancer metabolism. Cell Metab. 2016;23(1):27-47.

3. Boroughs LK, DeBerardinis RJ. Metabolic pathways promoting cancer cell survival and growth. Nat Cell Biol. 2015;17(4):351-359.

4. Kreuzaler P, et al. Adapt and conquer: metabolic flexibility in cancer growth, invasion and evasion. Mol Metab. 2020;33:83-101.

5. Hirayama A, et al. Quantitative metabolome profiling of colon and stomach cancer microenvironment by capillary electrophoresis time-of-flight mass spectrometry. Cancer Res. 2009;69(11):4918-4925.

6. Urasaki Y, et al. Coupling of glucose deprivation with impaired histone $\mathrm{H} 2 \mathrm{~B}$ monoubiquitination in tumors. PLoS One. 2012;7(5):e36775.

7. Vander Heiden MG, DeBerardinis RJ. Understanding the intersections between metabolism and cancer biology. Cell. 2017;168(4):657-669.

8 . Yun J, et al. Glucose deprivation contributes to the development of KRAS pathway mutations in tumor cells. Science. 2009;325(5947):1555-1559.

9. Miyo M, et al. Metabolic adaptation to nutritional stress in human colorectal cancer. Sci Rep. 2016;6:38415.

10. Zhang M, et al. Pim1 supports human colorectal cancer growth during glucose deprivation by enhancing the Warburg effect. Cancer Sci. 2018;109(5):1468-1479.

11. Ma L, et al. Control of nutrient stress-induced metabolic reprogramming by $\mathrm{PKC} \zeta$ in tumorigenesis. Cell. 2013;152(3):599-611.

12. Tang J, et al. LncRNA GLCC1 promotes colorectal carcinogenesis and glucose metabolism by stabilizing c-Myc. Nat Commun. 2019;10(1):3499.

13. Sanchez-Martinez R, et al. A link between lipid metabolism and epithelial-mesenchymal transition provides a target for colon cancer therapy. Oncotarget. 2015;6(36):38719-38736.

14. Morandi A, et al. Targeting the metabolic reprogramming that controls epithelial-to-mesenchymal transition in aggressive tumors. Front Oncol. 2017;7:40

15. Carracedo A, et al. Cancer metabolism: fatty acid oxidation in the limelight. Nat Rev Cancer.
2013;13(4):227-232.

16. Wang YN, et al. CPT1A-mediated fatty acid oxidation promotes colorectal cancer cell metastasis by inhibiting anoikis. Oncogene. 2018;37(46):6025-6040.

17. Wen YA, et al. Adipocytes activate mitochondrial fatty acid oxidation and autophagy to promote tumor growth in colon cancer. Cell Death Dis. 2017;8(2):e2593.

18. Taniguchi K, Karin M. NF- $\kappa$ B, inflammation, immunity and cancer: coming of age. Nat Rev Immunol. 2018;18(5):309-324.

19. DiDonato JA, et al. NF-kappa B and the link between inflammation and cancer. Immunol Rev. 2012;246(1):379-400.

20. Schwitalla S, et al. Intestinal tumorigenesis initiated by dedifferentiation and acquisition of stemcell-like properties. Cell. 2013;152(1-2):25-38.

21. Greten FR, et al. IKKbeta links inflammation and tumorigenesis in a mouse model of colitis-associated cancer. Cell. 2004;118(3):285-296.

22. Ishak Gabra MB, et al. IKKbeta activates p53 to promote cancer cell adaptation to glutamine deprivation. Oncogenesis. 2018;7(11):93.

23. Wang $X$, et al. $\alpha$-Ketoglutarate-activated NF- $\kappa B$ signaling promotes compensatory glucose uptake and brain tumor development. Mol Cell. 2019;76(1):148-162.

24. Mauro C, et al. NF- $\kappa$ B controls energy homeostasis and metabolic adaptation by upregulating mitochondrial respiration. Nat Cell Biol. 2011;13(10):1272-1279.

25. Maedera S, et al. GLUT6 is a lysosomal transporter that is regulated by inflammatory stimuli and modulates glycolysis in macrophages. FEBS Lett. 2019;593(2):195-208.

26. Bhat KPL, et al. Mesenchymal differentiation mediated by NF- $\mathrm{B}$ promotes radiation resistance in glioblastoma. Cancer Cell. 2013;24(3):331-346.

27. Wu Y, et al. Stabilization of snail by NF-kappaB is required for inflammation-induced cell migration and invasion. Cancer Cell. 2009;15(5):416-428.

28. Tornatore L, et al. The nuclear factor kappa B signaling pathway: integrating metabolism with inflammation. Trends Cell Biol. 2012;22(11):557-566.

29. Patel M, et al. NF-кB pathways in the develop- ment and progression of colorectal cancer. Transl Res. 2018;197:43-56.

30. Kojima M, et al. Increased nuclear factor-kB activation in human colorectal carcinoma and its correlation with tumor progression. Anticancer Res. 2004;24(2b):675-681.

31. Moorchung N, et al. An evaluation of nuclear factor kappa B expression in colorectal carcinoma: an analysis of 50 cases. J Cancer Res Ther. 2014;10(3):631-635.

32. Wu D, et al. NF- $\kappa \mathrm{B}$ expression and outcomes in solid tumors: a systematic review and meta-analysis. Medicine (Baltimore). 2015;94(40):e1687.

33. Guinney J, et al. The consensus molecular subtypes of colorectal cancer. Nat Med. 2015;21(11):1350-1356.

34. Hayden MS, Ghosh S. Signaling to NF-kappaB. Genes Dev. 2004;18(18):2195-2224.

35. Goracci L, et al. Lipostar, a comprehensive platform-neutral cheminformatics tool for lipidomics. Anal Chem. 2017;89(11):6257-6264.

36. Luo JL, et al. IKK/NF-kappaB signaling: balancing life and death--a new approach to cancer therapy. J Clin Invest. 2005;115(10):2625-2632.

37. Birsoy K, et al. Metabolic determinants of cancer cell sensitivity to glucose limitation and biguanides. Nature. 2014;508(7494):108-112.

38. Rambold AS, et al. Fatty acid trafficking in starved cells: regulation by lipid droplet lipolysis, autophagy, and mitochondrial fusion dynamics. Dev Cell. 2015;32(6):678-692.

39. Zhang L, Romero P. Metabolic control of CD8 $\mathrm{T}$ cell fate decisions and antitumor immunity. Trends Mol Med. 2018;24(1):30-48.

40. Lian J, et al. Carboxylesterases in lipid metabolism: from mouse to human. Protein Cell. 2018;9(2):178-195.

41. Dominguez E, et al. Integrated phenotypic and activity-based profiling links Ces3 to obesity and diabetes. Nat Chem Biol. 2014;10(2):113-121.

42. Gilham D, et al. Inhibitors of hepatic microsomal triacylglycerol hydrolase decrease very low density lipoprotein secretion. FASEB J. 2003;17(12):1685-1687.

43. Aflaki E, et al. Triacylglycerol accumulation activates the mitochondrial apoptosis pathway in macrophages. J Biol Chem. 2011;286(9):7418-7428.

44. Galluzzi L, et al. Molecular mechanisms of cell 
death: recommendations of the Nomenclature Committee on Cell Death 2018. Cell Death Differ. 2018;25(3):486-541.

45. Yang WS, Stockwell BR. Ferroptosis: death by lipid peroxidation. Trends Cell Biol. 2016;26(3):165-176.

46. Stockwell BR, et al. Ferroptosis: a regulated cell death nexus linking metabolism, redox biology, and disease. Cell. 2017;171(2):273-285.

47. Feng H, Stockwell BR. Unsolved mysteries: how does lipid peroxidation cause ferroptosis? PLOS Biol. 2018;16(5):e2006203.

48. Yang WS, et al. Regulation of ferroptotic cancer cell death by GPX4. Cell. 2014;156(1-2):317-331.

49. Cancer Genome Atlas Network. Comprehensive molecular characterization of human colon and rectal cancer. Nature. 2012;487(7407):330-337.

50. Calle EE, et al. Overweight, obesity, and mortality from cancer in a prospectively studied cohort of
U.S. adults. NEngl JMed. 2003;348(17):1625-1638.

51. O'Keefe SJD. Diet, microorganisms and their metabolites, and colon cancer. Nat Rev Gastroenterol Hepatol. 2016;13(12):691-706.

52. Park J, et al. Obesity and cancer - mechanisms underlying tumour progression and recurrence. Nat Rev Endocrinol. 2014;10(8):455-465.

53. Wang H, et al. Somatic gene copy number alterations in colorectal cancer: new quest for cancer drivers and biomarkers. Oncogene. 2016;35(16):2011-2019.

54. Xu J, et al. Carboxylesterase 1 Is regulated by hepatocyte nuclear factor $4 \alpha$ and protects agains alcohol- and MCD diet-induced liver injury. Sci Rep. 2016;6:24277.

55. Huang J, et al. Tumor-induced hyperlipidemia contributes to tumor growth. Cell Rep. 2016;15(2):336-348.

56. Nieman KM, et al. Adipose tissue and adipocytes support tumorigenesis and metastasis. Biochim Biophys Acta. 2013;1831(10):1533-1541.

57. Dienstmann R, et al. Consensus molecular subtypes and the evolution of precision medicine in colorectal cancer. Nat Rev Cancer. 2017;17(4):268.

58. Nieman KM, et al. Adipocytes promote ovarian cancer metastasis and provide energy for rapid tumor growth. Nat Med. 2011;17(11):1498-1503.

59. Counihan JL, et al. Cancer metabolism: current understanding and therapies. Chem Rev. 2018;118(14):6893-6923.

60. DeBerardinis RJ, Chandel NS. Fundamentals of cancer metabolism. Sci Adv. 2016;2(5):e1600200.

61. Wei E, et al. Loss of TGH/Ces3 in mice decreases blood lipids, improves glucose tolerance, and increases energy expenditure. Cell Metab. 2010;11(3):183-193. 